\title{
A slow pushed front in a Lotka-Volterra competition model
}

\author{
Matt Holzer and Arnd Scheel \\ University of Minnesota \\ School of Mathematics \\ 127 Vincent Hall, 206 Church St SE \\ Minneapolis, MN 55455, USA
}

May 11, 2012

\begin{abstract}
We study invasion speeds in the Lotka-Volterra competition model when the rate of diffusion of one species is small. Our main result is the construction of the selected front and a rigorous asymptotic approximation of its propagation speed, valid to second order. We use techniques from geometric singular perturbation theory and geometric desingularization. The main challenge arises from the slow passage through a saddle-node bifurcation. From a perspective of linear versus nonlinear speed selection, this front provides an interesting example as the propagation speed is slower than the linear spreading speed. However, our front shares many characteristics with pushed fronts that arise when the influence of nonlinearity leads to faster than linear speeds of propagation. We show that this is a result of the linear spreading speed arising as a simple pole of the resolvent instead of as a branch pole. Using the pointwise Green's function, we show that this pole poses no a priori obstacle to marginal stability of the nonlinear traveling front, thus explaining how nonlinear systems can exhibit slower spreading that their linearization in a robust fashion.
\end{abstract}

MSC numbers: 35C07, 35K57, 34E99

Keywords: invasion fronts, pushed and pulled fronts, Lotka-Volterra systems, pointwise Green's function

\section{Introduction}

We consider the problem of wavespeed selection in the diffusive Lotka-Volterra competition model

$$
\begin{aligned}
& u_{t}=\epsilon^{2} u_{x x}+\left(1-u-a_{1} v\right) u \\
& v_{t}=v_{x x}+r\left(1-a_{2} u-v\right) v .
\end{aligned}
$$

This model represents the interaction of two competing populations. We focus on the case where $r>0$, $a_{1}<1<a_{2}$ and $0<\epsilon \ll 1$. In this parameter regime, there exist three homogeneous steady states: a saddle $P_{-}=(0,1)$, a sink $P_{+}=(1,0)$ and the trivial unstable fixed point at the origin. In the absence of the $u$ species the system is expected to attain a uniform distribution of the $v$ species throughout the domain. This state is destabilized by the local introduction of a small amount of the $u$ species. This perturbation will grow and spread into the neighboring domain leaving behind the stable state wherein the $v$ species is absent. Of natural interest is the long time asymptotic behavior of this solution, supposing that the initial perturbation was compactly supported. Among the features of this invasion front that one would like to understand are the asymptotic front profile, the velocity with which this front propagates and the pattern that is left in the wake.

There is a vast literature concerning front propagation and selection. These studies fall into two loose groups. The first rely on comparison methods and date to the classical works of Fisher [12] and Kolmogorov, 
Petrovskii, and Piscounov [23]. These authors studied fronts in a scalar reaction-diffusion equation, now referred to as the Fisher-KPP equation, finding a continuum of possible wavespeeds and establishing a large class of initial conditions that converge to the slowest propagating front that remains everywhere positive. Central to this work is the existence of a comparison principle that provides some global nonlinear structure. For systems that lack this structure, a precise characterization of the basin of attraction of a particular traveling front is usually unattainable and other selection criteria must be used. Much of the literature for more general systems has been developed within the physics community. The focus of most of this work is determining necessary conditions for front selection. Notably, Dee and Langer [8] began the study of front selection in terms of stability properties of the traveling front. The selected front is classified by the property of marginal stability, which is to say that perturbations of the selected front neither grow nor decay when viewed pointwise in a frame moving with the speed of the front. These concepts have been applied and extended by a number of authors, notably van Saarloos, who has an excellent review on this topic [29].

Front propagation can be driven by linear or nonlinear effects and the terminology of pulled and pushed fronts has been developed to differentiate between these two cases. Pulled fronts are driven by the linear instability ahead of the front and propagate at the linear spreading speed. For general systems, computation of linear spreading speeds was first tackled within the plasma physics community with the goal of differentiating between absolute and convective instabilities, see $[4,2,8,19,29,28]$ for this work and subsequent extensions. Making use of a pointwise Green's function, the linear spreading speed can be computed explicitly; the details of this computation will be elucidated in section 4. It is worth noting that for systems of equations, individual components may propagate at different speeds. In this case, the largest of these speeds is the linear spreading speed.

Alternatively, the selected front may propagate at a speed different from the linear spreading speed. These pushed fronts are necessarily driven by the nonlinearity, usually due to the amplification or dampening of the linear instability by the nonlinearity. For scalar equations, when a pushed front exists it must travel faster than the linear spreading speed, hence the term pushed. Demonstrating that this is not always true for systems of equations is one of the aims of this paper and one of the reasons we became interested in the model (1.1). That a selected front, a pushed front in this case, could move slower than the linear spreading speed is somewhat surprising. One expects that a small perturbation of the front, placed far ahead of the front interface, should evolve essentially according to the (faster) linear dynamics. However, this is not always the case. A full explanation of this phenomena will be given in section 4 . There we differentiate between two different types of linear spreading: one arising due to a regular pole of the pointwise Green's function and one arising from a branch pole. The spreading speeds determined by the latter place a lower bound on the spreading speed of (1.1) while the former do not.

Finding pushed fronts requires a fully nonlinear treatment of (1.1). These fronts are stationary solutions in a moving coordinate frame $(\xi=x+s t)$, satisfying the system of second order differential equations,

$$
\begin{aligned}
& 0=\epsilon^{2} u_{\xi \xi}-s u_{\xi}+\left(1-u-a_{1} v\right) u \\
& 0=v_{\xi \xi}-s u_{\xi}+r\left(1-a_{2} u-v\right) v .
\end{aligned}
$$

Expand this system into a system of four first order equations. Nonlinear traveling fronts are found as heteroclinic connections between homogeneous states, in this case between $P_{-}$and $P_{+}$. A simple computation finds that the unstable manifold of $P_{-}$is three dimensional whereas the stable manifold of $P_{+}$is two dimensional. Dimension counting thus suggests that if a nonlinear front solution exists for some value of $s$, we would then expect an interval of $s$ values that will also give rise to a nonlinear traveling front.

To determine which, if any of these fronts are a pushed front we appeal to the marginal stability criterion of [8]. It is again useful to contrast with the case of scalar reaction-diffusion equations. Here the unstable manifold corresponding to the unstable homogeneous state is two dimensional. The decay rate of a nonlinear front is determined by the asymptotic behavior of the heteroclinic orbit as the unstable state is approached. Generically, such solutions will approach along the weakest eigendirection. Working in a weighted function space, an optimal exponential weight can be found that restricts the size and decay rates of perturbations allowed ahead of the front. The optimal weight discriminates against these weakly decaying fronts, requiring 
steeper decay for perturbations than for that of the front itself. This forces the expected zero eigenvalue out of the weighted function space. Now suppose that for some value of $s$ the approach is instead in the strong unstable manifold. The decay of this front is sufficiently steep so as this front still retains a zero eigenvalue in this weighted space. Thus, we see that the fronts with weak decay are stable and outrun any compactly supported perturbation ahead of the front. On the other hand, the strongly decaying front is only marginally stable and compactly supported perturbations will neither grow nor decay due to the projection onto the zero eigenfunction. Generalizing the steepest front criterion to systems requires some thought, as there are now more possible submanifolds for approach and it is not clear whether an optimal weight can be chosen. However, we expect that the marginal stability criterion should hold and that any pushed front will possess an embedded zero eigenvalue.

This brings us to our interest in the model (1.1). This model provides a tractable example in which to examine some of the theoretical issues that arise for front selection when dealing with systems of reactiondiffusion equations. The question of whether the selected front is linearly or nonlinearly determined for a variety of parameters in system (1.1) has received considerable interest in the literature, see for example $[16,25,17,18,14]$. Most closely related to this paper is the work of Hosono [17], who studies fronts in the parameter regime detailed above. There, asymptotic matching is used to construct nonlinear front solutions for all wavespeeds greater than $2 \epsilon \sqrt{1-a_{1} \beta}$ for some given value of $0<\beta<1$. It is conjectured that the slowest of these fronts provides a leading order approximation of the selected wavefront. Our results confirm this and we provide a rigorous next order correction to the selected wavespeed,

$$
s_{\text {crit }}(\epsilon)=2 \epsilon \sqrt{1-a_{1} \beta}-\frac{\Omega_{0} a_{1}^{2 / 3} \Gamma(\beta, r)^{2 / 3}}{\sqrt{1-a_{1} \beta}} \epsilon^{5 / 3}+\mathcal{O}\left(\epsilon^{2} \log (\epsilon)\right) .
$$

Our proof uses geometric singular perturbation theory $[11,20]$ to construct the selected front as the transverse intersection of manifolds. There are two main subtleties to the analysis. In contrast to the scalar equations discussed above, the selected front is not the steepest possible front and therefore determining the appropriate manifolds to use in this construction is non-trivial. The actual tracking of these manifolds is straight-forward, with the exception of one point where the analysis reduces, after several changes of variables, to a slow flow past a fold point. This complication is naturally resolved using geometric desingularization, [24]. Typical of problems of this form are correction terms of $\mathcal{O}\left(\epsilon^{2 / 3}\right)$. This explains the $\mathcal{O}\left(\epsilon^{5 / 3}\right)$ term in the wavespeed.

We claim that this front is the selected front and that it is a pushed front. To justify this, we show that this constructed front satisfies the marginal stability criterion with a simple zero eigenvalue at the origin, in a suitable weighted function space. Our approach makes use of a comparison principle for (1.1). We adapt a proof of Kan-on and Fang [21], which established the asymptotic stability of monotone fronts connecting stable homogeneous states (again making use of a comparison principle). We remark that since (1.1) satisfies a comparison principle, the selected front can be characterized as the slowest monotone front that remains everywhere positive, $[25,26]$. We confirm that the front constructed here satisfies these conditions. Namely, any front with slower speed is necessarily non-monotone. We emphasize that the existence of a comparison principle is not required for the observed phenomena. It is conceivable that marginal stability of this front could be established using geometric singular perturbation and geometric desingularization techniques. However, given that the machinery to prove marginal stability is already in place using comparison principles, we adopt that line of analysis here.

Finally, we show that this pushed front propagates slower than the linear spreading speed, thus demonstrating a novel phenomena. The linear spreading speed can be calculated and is $\mathcal{O}(1)$, much faster than the speed of the constructed front. We emphasize that the spreading speed (1.2) can not be determined by the linearization about the unstable state.

Our paper is organized as follows. In section 2 we prove the existence of the selected front. In section 3, we show that this front is asymptotically nonlinear stable in a weighted Banach space. In section 4, we compute the linear spreading speed and explain how the selected front is stable despite this fast linear spreading. We conclude with some remarks on general aspects of slower than linear spreading. 


\section{Existence of the pushed front}

In this section, we will prove the existence of a traveling front solution to (1.1). Let $\xi=x+\epsilon \sigma t$ and consider the system $(1.1)$ in $(t, \xi)$ coordinates,

$$
\begin{aligned}
u_{t} & =\epsilon^{2} u_{\xi \xi}-\epsilon \sigma u_{\xi}+\left(1-u-a_{1} v\right) u \\
v_{t} & =v_{\xi \xi}-\epsilon \sigma v_{\xi}+r\left(1-a_{2} u-v\right) v .
\end{aligned}
$$

We seek a stationary solution to these equations with asymptotic limits $P_{-}$and $P_{+}$as $\xi \rightarrow \pm \infty$. Stationary solutions are solutions of the system of second order ordinary differential equations,

$$
\begin{aligned}
& 0=\epsilon^{2} u_{\xi \xi}-\epsilon \sigma u_{\xi}+\left(1-u-a_{1} v\right) u \\
& 0=v_{\xi \xi}-\epsilon \sigma v_{\xi}+r\left(1-a_{2} u-v\right) v .
\end{aligned}
$$

We now state the main result of this paper. Before doing so, we introduce some notation. Suppose $X$ is a Banach Space of functions on $\mathbb{R}$ (for instance $C_{\text {unif }}(\mathbb{R})$ ). Let

$$
W(\xi, \epsilon)=1+e^{\frac{-2 \sqrt{1-a_{1}}}{\epsilon} \xi} .
$$

Then define the weighted space by $X_{W}:=\{u \in X \mid u(t, \cdot) W(\cdot) \in X\}$.

Theorem 1. Suppose $r>0$ and $a_{1}<1<a_{2}$. Then there exists $\epsilon_{0}>0$ such that for all $0<\epsilon<\epsilon_{0}$, there exists a locally unique wave speed $\sigma_{\text {crit }}(\epsilon)$ such that system (1.1) has a monotone, positive traveling front solution with $(U(\xi), V(\xi)) \in X_{W} \times X$ satisfying $(U, V) \rightarrow P_{-}$as $\xi \rightarrow-\infty$ and $(U, V) \rightarrow P_{+}$as $\xi \rightarrow \infty$. Furthermore,

$$
\sigma_{\text {crit }}(\epsilon)=2 \sqrt{1-a_{1} \beta}-\frac{\Omega_{0} a_{1}^{2 / 3} \Gamma(\beta, r)^{2 / 3}}{\sqrt{1-a_{1} \beta}} \epsilon^{2 / 3}+\mathcal{O}(\epsilon \log (\epsilon)) .
$$

The above quantities will be described later in detail, but we mention them here for completeness: $\beta$ is the unique zero of the cubic $\frac{2 a_{1} a_{2}}{3} v^{3}-a_{2} v^{2}+\frac{1}{3}=0$ in the unit interval, $\Gamma(\beta, r)=\sqrt{\frac{r}{3}-r \beta^{2}+\frac{2}{3} r \beta^{3}}$ and $\Omega_{0} \approx 2.3381$ is the smallest positive zero of the expression,

$$
J_{-1 / 3}\left(2 z^{3 / 2} / 3\right)+J_{1 / 3}\left(2 z^{3 / 2} / 3\right),
$$

with $J_{ \pm 1 / 3}$ Bessel functions of the first kind.

We will outline our strategy for the proof of Theorem 1 below, but for now focus on the necessity of restricting to the weighted Banach space, $X_{W} \times X$. Expanding (2.2) into a system of first order equations we have,

$$
\begin{aligned}
\epsilon u_{1}^{\prime} & =u_{2} \\
\epsilon u_{2}^{\prime} & =\sigma u_{2}-\left(1-u_{1}-a_{1} v_{1}\right) u_{1}, \\
v_{1}^{\prime} & =v_{2} \\
v_{2}^{\prime} & =\epsilon \sigma v_{2}-r\left(1-a_{2} u_{1}-v_{1}\right) v_{1} .
\end{aligned}
$$

A traveling front is a heteroclinic orbit connecting the fixed point $P_{-}=(0,0,1,0)^{T}$ to $P_{+}=(1,0,0,0)^{T}$. This heteroclinic orbit must lie in both the stable manifold of $P_{+}$and the unstable manifold of $P_{-}$. Due to the invariance of the axes, the eigenvalues of $P_{+}$and $P_{-}$can be computed exactly. For $P_{+}$we find these eigenvalues to be,

$$
\nu_{1}^{ \pm}:=\frac{\sigma}{2 \epsilon} \pm \frac{1}{2 \epsilon} \sqrt{\sigma^{2}+4}, \quad \nu_{2}^{ \pm}:=\frac{\epsilon \sigma}{2} \pm \frac{1}{2} \sqrt{\epsilon^{2} \sigma^{2}+4 r\left(a_{2}-1\right)},
$$

with eigenvectors,

$$
n_{1}^{ \pm}:=\left(1, \epsilon \nu_{1}^{ \pm}, 0,0\right)^{T}, \quad n_{2}^{ \pm}:=\left(a_{1}, \epsilon a_{1} \nu_{2}^{ \pm}, N_{ \pm}, N_{ \pm} \nu_{2}^{ \pm}\right)^{T},
$$


with $N_{ \pm}=-1-\epsilon \sigma \nu_{2}^{ \pm}+\left(\epsilon \nu_{2}^{ \pm}\right)^{2}$. For $P_{-}$we have

$$
\mu_{1}^{ \pm}:=\frac{\sigma}{2 \epsilon} \pm \frac{1}{2 \epsilon} \sqrt{\sigma^{2}-4\left(1-a_{1}\right)}, \quad \mu_{2}^{ \pm}:=\frac{\epsilon \sigma}{2} \pm \frac{1}{2} \sqrt{\epsilon^{2} \sigma^{2}+4 r},
$$

with eigenvectors,

$$
m_{1}^{ \pm}:=\left(M_{ \pm}, M_{ \pm} \mu_{1}^{ \pm}, r a_{2}, r a_{2} \mu_{1}^{ \pm}\right)^{T}, \quad m_{2}^{ \pm}:=\left(0,0,1, \mu_{2}^{ \pm}\right)^{T},
$$

with $M_{ \pm}=\left(\mu_{1}^{ \pm}\right)^{2}-\epsilon \sigma \mu_{1}^{ \pm}-r$. Given our choice of parameters, it is easy to see that the fixed point at $P_{-}$has a three dimensional unstable manifold whereas $P_{+}$has a two dimensional stable manifold. To select a unique front, we require that the heteroclinic solution actually lies in a particular two dimensional sub-manifold of the unstable manifold.

We denote this sub-manifold $W^{u u}\left(P_{-}\right)$, with definition,

$$
W^{u u}\left(P_{-}\right):=\left\{\left(u_{1}, u_{2}, v_{1}, v_{2}\right) \in W^{u}\left(P_{-}\right) \mid \Phi\left(\xi, u_{1}, u_{2}, v_{1}, v_{2}\right) \in X_{w}^{2} \times X^{2} \quad \text { for } \quad \xi \rightarrow-\infty\right\},
$$

where $\Phi$ is the flow associated to (2.4).

We now turn to the proof of Theorem 1 . This proof will use techniques from geometric singular perturbation theory $[11,20]$. We will exploit the separation of time scales induced by the small diffusion constant $\epsilon^{2}$. The basic approach is as follows. The manifolds $W^{u u}\left(P_{-}\right)$and $W^{s}\left(P_{+}\right)$depend on the wavespeed $\sigma$. We append a trivial equation for $\sigma$ and look for intersections of these, now three dimensional manifolds, in five dimensional space. We first study the $\epsilon=0$ problem, constructing a singular solution from concatenated pieces of fixed points and solutions to this reduced problem (2.4). We then track $W^{u u}\left(P_{-}\right)$and $W^{s}\left(P_{+}\right)$ along this singular solution and show that they intersect transversely. Since these manifolds vary smoothly with $\epsilon$, this transverse intersection will imply the existence of a nearby solution to the $\epsilon \neq 0$ problem. This will establish Theorem 1.

\subsection{Fast-Slow Structure}

When $\epsilon=0$ in (2.4), the system is diffeo-algebraic. The slow manifold is the set of $(u, v)$ so that $(1-u-$ $\left.a_{1} v\right) u=0$. Thus, the slow manifold is given by the union of two manifolds. Let,

$$
\mathcal{M}_{L, 0}:=\left\{\left(u_{1}, u_{2}, v_{1}, v_{2}\right) \mid u_{1}=0, u_{2}=0\right\}, \quad \mathcal{M}_{R, 0}:=\left\{\left(u_{1}, u_{2}, v_{1}, v_{2}\right) \mid u_{1}=1-a_{1} v_{1}, u_{2}=0\right\} .
$$

The reduced dynamics governing the slow flow on the left slow manifold, $\mathcal{M}_{L, 0}$, are given by

$$
\begin{aligned}
& v_{1}^{\prime}=v_{2} \\
& v_{2}^{\prime}=-r\left(1-v_{1}\right) v_{1} .
\end{aligned}
$$

This system is Hamiltonian with

$$
H_{L}\left(v_{1}, v_{2}\right)=\frac{v_{2}^{2}}{2}+\frac{r v_{1}^{2}}{2}-\frac{r v_{1}^{3}}{3} .
$$

The level set $H_{L}\left(v_{1}, v_{2}\right)=\frac{r}{6}$ corresponds to those orbits in the slow manifold that are backwards or forwards asymptotic to the fixed point $P_{-}$. Alternatively, the reduced equations on, $\mathcal{M}_{R, 0}$, are

$$
\begin{aligned}
& v_{1}^{\prime}=v_{2} \\
& v_{2}^{\prime}=-r\left(1-a_{2}+a_{1} a_{2} v_{1}-v_{1}\right) v_{1} .
\end{aligned}
$$

Again, this system is Hamiltonian with

$$
H_{R}\left(v_{1}, v_{2}\right)=\frac{v_{2}^{2}}{2}+\frac{r v_{1}^{2}}{2}-\frac{r a_{2} v_{1}^{2}}{2}+\frac{r a_{1} a_{2} v_{1}^{3}}{3}-\frac{r v_{1}^{3}}{3} .
$$


The level set $H_{R}\left(v_{1}, v_{2}\right)=0$ corresponds to those orbits in $\mathcal{M}_{R, 0}$ that are either backward or forward asymptotic to the fixed point $P_{+}$. These level curves intersect at $\left(v_{1}, v_{2}\right)=(\beta,-\Gamma(\beta, r))$, where $\beta$ is the unique solution in the unit interval of $Q\left(v_{1}\right)=0$, with,

$$
Q\left(v_{1}\right):=\frac{r a_{1} a_{2}}{3} v_{1}^{3}-\frac{r a_{2}}{2} v_{1}^{2}+\frac{r}{6},
$$

and $\Gamma(\beta, r)$ satisfies,

$$
\Gamma(\beta, r)=\sqrt{\frac{r}{3}-r \beta^{2}+\frac{2}{3} r \beta^{3}} .
$$

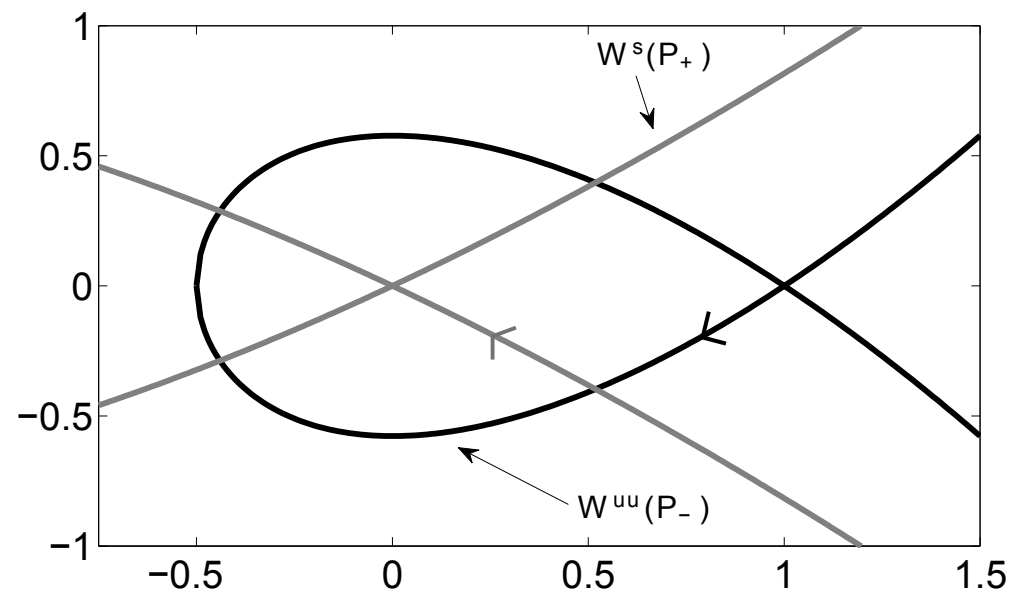

Figure 1: The reduced dynamics on $\mathcal{M}_{L, 0}$ and $\mathcal{M}_{R, 0}$, projected onto the $v_{1}-v_{2}$ plane. Here the black curves are a level set of $H_{L}\left(v_{1}, v_{2}\right)$ giving the dynamics of the unstable manifold of $P_{-}$in equation (2.9). The gray curves are the zero level set of $H_{R}\left(v_{1}, v_{2}\right)$, giving the stable manifold of $P_{+}$in $\mathcal{M}_{R, 0}$, evolving under (2.10). For $v_{1}=\beta$, these level sets intersect at two values of $v_{2}$. The intersection with negative $v_{2}$ pertains to fronts propagating to the left.

See Figure 1. For $\left(v_{1}, v_{2}\right)=(\beta,-\Gamma(\beta, r))$, we will show in the next section that there exists a singular fast connection between $\mathcal{M}_{L, 0}$ and $\mathcal{M}_{R, 0}$. Before doing so, we make some observations concerning these slow manifolds.

Away from their intersection at $v_{1}=1 / a_{1}$, both $\mathcal{M}_{L, 0}$ and $\mathcal{M}_{R, 0}$ are normally hyperbolic slow manifolds which persist as such for $0<\epsilon \ll 1$. In particular, we have

Lemma 2.1. [11] For $v_{1}<\frac{1}{a_{1}}$, there exists smooth manifolds, $\mathcal{M}_{L, \epsilon}$ and $\mathcal{M}_{R, \epsilon}, C^{1}, \mathcal{O}(\epsilon)$ close to $\mathcal{M}_{L, 0}$ and $\mathcal{M}_{R, 0}$ respectively. Due to the invariance of the $u_{1}=0, u_{2}=0$ subspace, we can select $\mathcal{M}_{L, \epsilon}:=$ $\left\{\left(u_{1}, u_{2}, v_{1}, v_{2}\right) \mid u_{1}=0, u_{2}=0\right\}$. In addition, $\mathcal{M}_{L, \epsilon}$ has a smooth four dimensional unstable manifold comprised of the manifold itself and the union of its unstable fibers, while $\mathcal{M}_{R, \epsilon}$ has both a three dimensional smooth unstable manifold and three dimensional smooth stable manifold.

The unstable manifold of $\mathcal{M}_{L, \epsilon}$ can be further resolved by noting that the eigenvalues transverse to the slow manifold are

$$
\frac{\sigma}{2 \epsilon} \pm \frac{1}{2 \epsilon} \sqrt{\sigma^{2}-4\left(1-a_{1} v_{1}\right)}
$$

and therefore when $\sigma>2 \sqrt{1-a_{1} v_{1}}$ there exists a spectral gap between these eigenvalues. With the aid of this spectral gap there exists an invariant family of one dimensional strong-unstable fibers, $\mathcal{F}^{u u}(p)$. These 
fibers are $\mathcal{O}(\epsilon)$ perturbations of the strong unstable manifold at $\epsilon=0$ (see [11]). This affords us the following description of the strong unstable manifold,

$$
W^{u u}\left(P_{-}\right)=\bigcup_{p \in \mathcal{M}_{L, \epsilon} \bigcap W^{u}\left(P_{-}\right)} \mathcal{F}^{u u}(p) .
$$

Of course, this decomposition is valid only so long as $\sigma>2 \sqrt{1-a_{1} v_{1}}$.

We now turn our attention to finding a singular connection between $\mathcal{M}_{L, 0}$ and $\mathcal{M}_{R, 0}$. This will provide a leading order estimate for $\sigma$.

\subsection{Leading order fast transition}

We return to the construction of the singular orbit. The singular orbit will consist of fixed points in the two slow manifolds, $\mathcal{M}_{L, 0}$ and $\mathcal{M}_{R, 0}$, as well as fast connection between these manifolds that we will describe presently.

The fast subsystem is found via the rescaling $\tau=\frac{\xi}{\epsilon}$ in $(2.4)$,

$$
\begin{aligned}
& \dot{u}_{1}=u_{2} \\
& \dot{u}_{2}=\sigma u_{2}-u_{1}\left(1-a_{1} v_{1}-u_{1}\right) \\
& \dot{v}_{1}=\epsilon v_{2} \\
& \dot{v}_{2}=\epsilon^{2} \sigma v_{2}-\epsilon r v_{1}\left(1-a_{2} u_{1}-v_{1}\right) .
\end{aligned}
$$

When $\epsilon=0$, the $v_{1}$ and $v_{2}$ coordinates act as parameters and the dynamics of the fast subsystem are governed by the scalar equation,

$$
\ddot{u}-\sigma \dot{u}+u\left(1-a_{1} v-u\right)=0 .
$$

Therefore, finding a fast transition that connects $\mathcal{M}_{L, 0}$ and $\mathcal{M}_{R, 0}$ is equivalent to finding traveling front solutions of the Fisher-KPP equation,

$$
u_{t}=u_{x x}+f(u, v), \quad f(u, v)=u\left(1-a_{1} v-u\right)
$$

The dynamics of this equation have been studied in great detail in many different contexts and are well understood $[12,23,1]$. For each fixed value of $v$ near $\beta$, we have $f(0, v)=f\left(1-a_{1} v, v\right)=0$ and $f(u, v)>0$ for all $0<u<1-a_{1} v$. Therefore, by [1] it is known that there exists a critical wave speed, $\sigma_{K P P}(v)$ such that (2.15) has a monotonic traveling front solution, $u(\tau)$ which is asymptotic to $\left(u_{1}, u_{2}\right)=(0,0)$ as $\tau \rightarrow-\infty$ and asymptotic to $\left(u_{1}, u_{2}\right)=\left(1-a_{1} v, 0\right)$ as $\tau \rightarrow \infty$ for any value of $\sigma>\sigma_{K P P}(v)$ with $\sigma_{K P P}(v)=2 \sqrt{f^{\prime}(0, v)}$. In addition, for $\sigma=\sigma_{K P P}(v)$, we have the following asymptotic characterization of the solution,

$$
u(\tau)=(A+B \tau) e^{\frac{\sigma_{K P P}}{2}}+\mathcal{O}\left(\tau^{2} e^{\sigma_{K P P} \tau}\right) \quad \text { as } \quad \tau \rightarrow-\infty,
$$

with $B<0$. In particular, for $v=\beta$ there exists a monotonic connection between $\mathcal{M}_{L, 0}$ and $\mathcal{M}_{R, 0}$ for all $\sigma \geq 2 \sqrt{1-a_{1} \beta}$. When $\sigma<2 \sqrt{1-a_{1} \beta}$, a connection persists, but is not monotonic. Since it will be of importance through this paper, we denote

$$
\sigma^{*}=2 \sqrt{1-a_{1} \beta} .
$$

The singular orbit is comprised of three pieces, see Figure 2:

1. The unstable manifold of $P_{-}$lying in $\mathcal{M}_{L, 0}$ for $\beta<v<1$

2. The critical F-KPP front connecting $\mathcal{M}_{L, 0}$ and $\mathcal{M}_{R, 0}$ at $v=\beta$

3. The stable manifold of $P_{+}$lying in $\mathcal{M}_{R, 0}$ for $0<v<\beta$. 


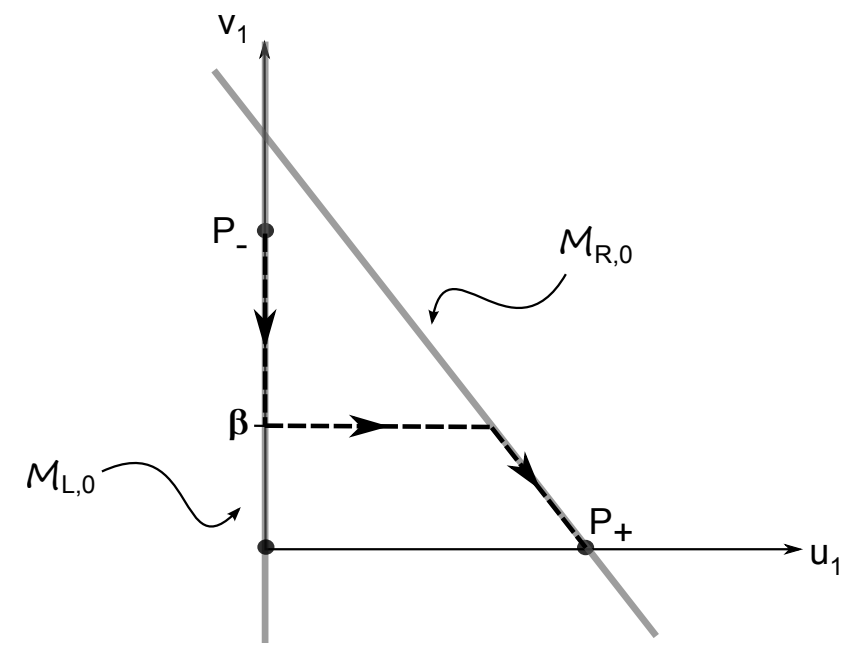

Figure 2: The dynamics of (2.14), plotted in configuration space. The dashed line is the singular solution, which jumps between the slow manifolds $\mathcal{M}_{L, 0}$ and $\mathcal{M}_{R, 0}$ at $v_{1}=\beta$.

Remark 1. The singular orbit could also be constructed with any of the family of front solutions for the $F-K P P$ equation at $\left(v_{1}, v_{2}\right)=(\beta,-\Gamma(\beta, r))$. We explain our rationale for restricting to the critical front. If we were to select a front corresponding to $\sigma>\sigma^{*}$, then we would be in the regime where the eigenvalues transverse to $\mathcal{M}_{L, 0}$ have a spectral gap. Since the F-KPP front for these wavespeeds depart $\mathcal{M}_{L, 0}$ along the weaker of these transverse eigendirections we see that the singular solution in this case would not lie in $W^{u u}\left(P_{-}\right) \cap W^{s}\left(P_{+}\right)$.

We now turn our attention to tracking $W^{u u}\left(P_{-}\right)$and $W^{s}\left(P_{+}\right)$along this singular solution.

\subsection{Geometric Desingularization}

Tracking the manifold $W^{u u}\left(P_{-}\right)$forward in $\xi$ will be the primary objective of this section. As we observed above, when there exists a spectral gap between the eigenvalues transverse to $\mathcal{M}_{L, \epsilon}$ then Fenichel Theory naturally gives $W^{u u}\left(P_{-}\right)$as a union of strong-unstable fibers. Subtleties arise when this spectral gap vanishes. Recall that the leading order wavespeed is given by $\sigma=2 \sqrt{1-a_{1} \beta}$. The transverse eigenvalues are given to leading order by $(2.12)$ and we note that exactly as $v_{1}$ reaches the transition point at $v_{1}=\beta$, the unstable eigenvalues that govern the dynamics transverse to the slow manifold coalesce. For $v_{1}<\beta$ these eigenvalues are complex conjugate and therefore in a neighborhood of $v_{1}=\beta$, local decay rates alone are an insufficient tool to characterize the strong unstable manifold. Instead, we will rely on geometric desingularization to track this manifold as it evolves into the oscillatory regime near $v_{1}=\beta$.

We perform several changes of variables to illuminate the underlying geometry.

Straightening the flow on $\mathcal{M}_{L, \epsilon}$. In a neighborhood of $\left(v_{1}, v_{2}\right)=(\beta,-\Gamma(\beta, r))$, the slow manifold $\mathcal{M}_{L, \epsilon}$ is normally hyperbolic and has no critical points for $\epsilon>0$. As a result, there exists a $\delta_{R}>0$ such that for all $\left|\left(v_{1}, v_{2}\right)-(\beta,-\Gamma(\beta, r))\right| \leq \delta_{R}$, there is a smooth invertible change of coordinates $(x, y)=\tilde{\Psi}_{1}\left(v_{1}, v_{2}\right)$, $(0,0)=\tilde{\Psi}_{1}(\beta,-\Gamma(\beta, r))$ that rectifies the slow flow along $\mathcal{M}_{L, \epsilon}$. Due to the Hamiltonian structure on $\mathcal{M}_{L, 0}$, we have

$$
\left(\begin{array}{c}
x \\
y
\end{array}\right)=\left(\begin{array}{c}
H_{L}\left(v_{1}, v_{2}\right)-\frac{r}{6}+\mathcal{O}(\epsilon) \\
\frac{v_{1}-\beta}{\Gamma(\beta, r)}+\mathcal{O}\left(\left(v_{1}-\beta\right)^{2}, \epsilon\right)
\end{array}\right) \text {. }
$$


In these new coordinates,

$$
\begin{aligned}
\dot{x} & =\epsilon g_{1}\left(x, y, u_{1}, u_{2}, \sigma, \epsilon\right) \\
\dot{y} & =-\epsilon\left(1+g_{2}\left(x, y, u_{1}, u_{2}, \sigma, \epsilon\right)\right) \\
\dot{u_{1}} & =u_{2} \\
\dot{u_{2}} & =\sigma u_{2}-\left(1-u_{1}-a_{1} \Gamma(\beta, r) y-a_{1} \beta+\mathcal{O}\left(y^{2}, \epsilon\right)\right) u_{1} .
\end{aligned}
$$

Here $g_{1}$ and $g_{2}$ are smooth functions satisfying $g_{1}(x, y, 0,0, \sigma, \epsilon)=0=g_{2}(x, y, 0,0, \sigma, \epsilon)$. Through an $\mathcal{O}(\epsilon)$ rescaling of $x$, we may choose $x=0$ to correspond to the unique orbit in $\mathcal{M}_{L, \epsilon}$ that converges to $P_{-}$as $\xi \rightarrow-\infty$. We denote the overall change of coordinates $\Psi_{1}\left(u_{1}, u_{2}, v_{1}, v_{2}, \sigma, \epsilon\right)=\left(u_{1}, u_{2}, \tilde{\Psi}_{1}^{1}, \tilde{\Psi}_{1}^{1}, \sigma, \epsilon\right)$.

Projective Coordinates (Blow-up of $\mathcal{M}_{L, \epsilon}$ ). As $y$ approaches zero, we noted above that the eigenvalues (and eigenvectors) transverse to $\mathcal{M}_{L, 0}$ coalesce. Converting the fast variables to polar coordinates $(\rho, \theta)$ maps each invariant line in $\mathcal{M}_{L, 0}$ ( $x$ equal to a constant, $y$ in some interval) to an invariant cylinder $(\rho=0, \theta \in[0,2 \pi))$. With $\epsilon=0$, for each fixed $y>0$ there exists four fixed points on the surface of the cylinder, corresponding to the weak-unstable and strong-unstable transverse eigenspaces. At $y=0$ these fixed points disappear via a saddle-node bifurcation. Tracking the evolution of points on the surface of this cylinder will allow us to track the unstable and strong-unstable eigenspaces into the oscillatory regime.

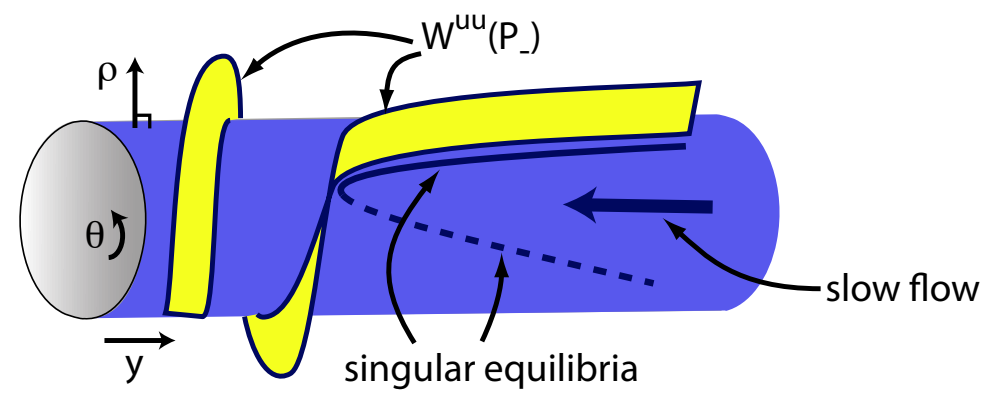

Figure 3: A schematic of the blown-up dynamics of (2.18). Here we fix $x=0$ and restrict to three dimensions. The invariant line with $x, u_{1}$ and $u_{2}$ all zero has been blown up to an invariant cylinder. Nontrivial dynamics are retained on the surface of this cylinder. The arrow denotes the direction of the slow flow in $y$. For $\epsilon=0$ and $y>0$, we see two fixed curves of singular equilibria, one stable and corresponding to the strong unstable eigendirection (shown as the solid black curve) and one unstable corresponding to the weak unstable eigendirection (the dashed black curve). At $y=0$, these fixed points disappear via a saddle-node bifurcation and for $y<0$ the flow winds around the cylinder, as does the tracked manifold.

Instead of polar coordinates, $(\rho, \theta)$ it is more convenient to use projective coordinates, $\left(u_{1}, z\right)$, with

$$
z=\frac{u_{2}}{u_{1}}
$$

to desingularize the flow transverse to the slow manifold. The $u_{1}$ coordinate measures the distance from the cylinder in the fast direction, while $z=\tan \theta$ is a measure of the angle around the cylinder. The coalescence of the transverse eigenvalues leads to behavior much like a saddle-node bifurcation of real subspaces. Using projective coordinates, this bifurcation structure is made explicit in the dynamics of the $z$ variable.

Using the change of coordinates

$$
\Psi_{2}:\left(x, y, u_{1}, u_{2}, \sigma, \epsilon\right) \rightarrow\left(x, y, u_{1}, z, \sigma, \epsilon\right)
$$


the equations in these coordinates become,

$$
\begin{aligned}
\dot{x} & =\epsilon \hat{g}_{1}\left(x, y, u_{1}, z, \eta, \epsilon\right) \\
\dot{y} & =-\epsilon\left(1+\hat{g}_{2}\left(x, y, u_{1}, z, \eta, \epsilon\right)\right) \\
\dot{u_{1}} & =z u_{1} \\
\dot{z} & =\sigma z-1+u_{1}+a_{1} \Gamma(\beta, r) y+a_{1} \beta-z^{2}+\mathcal{O}\left(y^{2}, \epsilon\right) \\
\dot{\epsilon} & =0 .
\end{aligned}
$$

We have appended a trivial equation for $\epsilon$ so that the system has a fixed point at $\left(x, y, u_{1}, z, \epsilon\right)=\left(x, 0,0, \frac{\sigma^{*}}{2}, 0\right)$. Since we are interested in values of $\sigma$ near $\sigma^{*}$ we introduce $\eta=\sigma-\sigma^{*}$, and make the rescalings

$$
\begin{aligned}
& \hat{z}=-z+\frac{\sigma^{*}+\eta}{2} \\
& \hat{y}=a_{1} \Gamma(\beta, r) y+\frac{\eta \sigma^{*}}{2}+\frac{\eta^{2}}{4} . \\
& \hat{\epsilon}=\epsilon a_{1} \Gamma(\beta, r) .
\end{aligned}
$$

We denote this change of coordinates by $\Psi_{3}:(x, y, u, z, \sigma, \epsilon) \rightarrow(x, \hat{y}, u, \hat{z}, \eta, \hat{\epsilon})$. Then,

$$
\begin{aligned}
\dot{x} & =\hat{\epsilon} \hat{g}_{1}\left(x, y, u_{1}, z, \eta, \hat{\epsilon}\right) \\
\dot{\hat{y}} & =-\hat{\epsilon}\left(1+\hat{g}_{2}\left(x, y, u_{1}, z, \eta, \hat{\epsilon}\right)\right) \\
\dot{u_{1}} & =\frac{\sigma^{*}}{2} u_{1}-\hat{z} u_{1}+\frac{\eta u_{1}}{2} \\
\dot{\hat{z}} & =\hat{z}^{2}-\hat{y}-u_{1}+\mathcal{O}\left(\hat{\epsilon}, y^{2}\right) \\
\dot{\eta} & =0 \\
\dot{\hat{\epsilon}} & =0 .
\end{aligned}
$$

For convenience, we will omit the subscript in $u_{1}$ in what follows.

\section{Center Manifold Reduction.}

Let $X=(x, \hat{y}, u, \hat{z}, \eta, \hat{\epsilon})$ and write the system (2.19) in the compact form,

$$
\dot{X}=A X+f(X),
$$

where $f(0)=0$ and $D f(0)=0$. Let $\phi\left(\tau, X_{0}\right)$ denote the flow associated to this system. For future reference,

$$
f(X)=\left(\hat{\epsilon} \hat{g_{1}}(X),-\hat{\epsilon} \hat{g_{2}}(X), f_{3}(X), f_{4}(X), 0,0\right)^{T} .
$$

Center Manifold Theory $[5,6]$ provides the following characterization of the flow in a neighborhood of the origin.

Lemma 2.2. The matrix A has five zero eigenvalues and one unstable eigenvalue, $\mu_{u}=\frac{\sigma^{*}}{2}$ with corresponding unstable eigenvector,

$$
\chi_{u}=\left(0,0, \sqrt{1-a_{1} \beta},-1,0,0\right)^{T} .
$$

There exists an open neighborhood of the origin, $\Omega$, with radius $\delta_{\Omega}<\delta_{R}$, so that $W_{\text {loc }}^{c}(0)=\{(x, \hat{y}, u, \hat{z}, \eta, \hat{\epsilon}) \mid u=$ $0\}$ is a smooth, five dimensional local center manifold. This manifold has an associated locally invariant unstable foliation $\Omega=\cup_{X \in W_{\text {loc }}^{c}(0)} M_{u}(X)$, where $M_{u}(X)$ is the one-dimensional unstable fiber associated to $X \in \Omega$. For this system, we have,

- $M_{u}(X)=\{X+J(X, c) \mid c \in \mathbb{R}\}$ with $J(X, c): \mathbb{R}^{6} \times \mathbb{R} \rightarrow \mathbb{R}^{6}$, uniformly continuous in $(X, c)$, a $C^{k}$ mapping of $\mathbb{R} \rightarrow \mathbb{R}^{6}$ for all $X \in \mathbb{R}^{6}$ and at least $C^{1}$ as a function of the basepoint $X$. 
- The function $J(X, c)$ can be decomposed into,

$$
J(X, c)=c \chi_{u}+J_{0}(X, c)+\hat{\epsilon} J_{1}(X, c),
$$

such that $\partial_{\hat{\epsilon}} J_{0}=0$, with constants $C_{0}$ and $C_{1}$ such that $\left\|D^{m} J_{0}(X, c)\right\|<C_{0} \delta_{\Omega}$ and $\left\|D^{m} J_{1}(X, c)\right\|<C_{1}$ for multi-indicies $m$ with $|m|=0,1$. In addition,

$$
J_{0}=\left(0,0, J_{0}^{(3)}, J_{0}^{(4)}, 0,0\right)^{T}, \quad J_{1}=\left(J_{1}^{(1)}, J_{1}^{(2)}, J_{1}^{(3)}, J_{1}^{(4)}, 0,0\right)^{T} .
$$

\section{Analysis of the flow in the center manifold.}

For $u=0$ and $\epsilon=0$, i.e. on the surface of the cylinder in the singular limit, we find that for $\hat{y}>0$, there are two fixed points given by the roots of the quadratic $\hat{y}=\hat{z}^{2}$. The negative root is stable and corresponds to the basepoint of tracked strong unstable manifold. Since the fiber attached to this basepoint is one dimensional there is no obstacle to tracking the strong unstable manifold into the regime $v<\beta$. We simply track the basepoint in the center manifold and due to the invariance of the family of unstable fibers, the corresponding unstable fiber is the tracked manifold. Therefore, we restrict our attention to the center manifold, where the reduced dynamics are,

$$
\begin{aligned}
\dot{x} & =0 \\
\dot{\hat{y}} & =-\hat{\epsilon} \\
\dot{\hat{z}} & =\hat{z}^{2}-\hat{y}+\mathcal{O}\left(\hat{\epsilon}, y^{2}\right) \\
\dot{\eta} & =0 \\
\dot{\hat{\epsilon}} & =0 .
\end{aligned}
$$

This system has a familiar form, namely it is the generic form of slow flow past a fold point studied in [24], albeit with the wavespeed and $x$ as additional (trivial) variables. The dynamics are well understood for sufficiently small, positive $\epsilon$.

Theorem 2. [24] Let $\delta>0$. Define the sections,

$$
\begin{aligned}
\Delta^{i n} & =\left\{(x, \hat{y}, u, \hat{z}, \eta, \hat{\epsilon}) \mid \quad u=0 \quad \hat{y}=\delta^{2}\right\} \\
\Delta^{\text {out }} & =\{(x, \hat{y}, u, \hat{z}, \eta, \hat{\epsilon}) \mid \quad u=0 \quad \hat{z}=\delta\} .
\end{aligned}
$$

The flow between these sections is described by equations (2.21). Let $\pi: \Delta^{i n} \rightarrow \Delta^{\text {out }}$ be the transition map for this flow. Let $p^{i n}=\left(x, \delta^{2}, 0,-\delta, \eta, \hat{\epsilon}\right)$. Then for all $\hat{\epsilon}$ sufficiently small, $\pi\left(p^{i n}\right)=(x,-h(\hat{\epsilon}), 0, \delta, \eta, \hat{\epsilon})$ where

$$
h(\hat{\epsilon})=-\Omega_{0} \hat{\epsilon}^{2 / 3}+\mathcal{O}(\hat{\epsilon} \log (\hat{\epsilon})) .
$$

In addition, for fixed $x, \eta$ and $\epsilon$, the transition map is a contraction as a map from $\hat{z}$ to $\hat{y}$ with contraction constant $\mathcal{O}\left(e^{-\frac{c}{\epsilon}}\right)$.

Here, $\Omega_{0}$ is the smallest positive zero of the expression,

$$
J_{-1 / 3}\left(2 z^{3 / 2} / 3\right)+J_{1 / 3}\left(2 z^{3 / 2} / 3\right),
$$

with $J_{ \pm 1 / 3}$ Bessel functions of the first kind (see [24]). See Figure 4.

We have now established all of the geometric information that we will need to prove Theorem 1.

\subsection{Proof of Theorem 1}

In this section, we use the geometric picture developed in the previous section to establish the transverse intersection of the manifolds $W^{u u}\left(P_{-}\right)$and $W^{s}\left(P_{+}\right)$. This will establish Theorem 1 . In the course of the proof, we will derive the asymptotic expansion for the selected wavespeed $\sigma_{\text {crit }}$. 


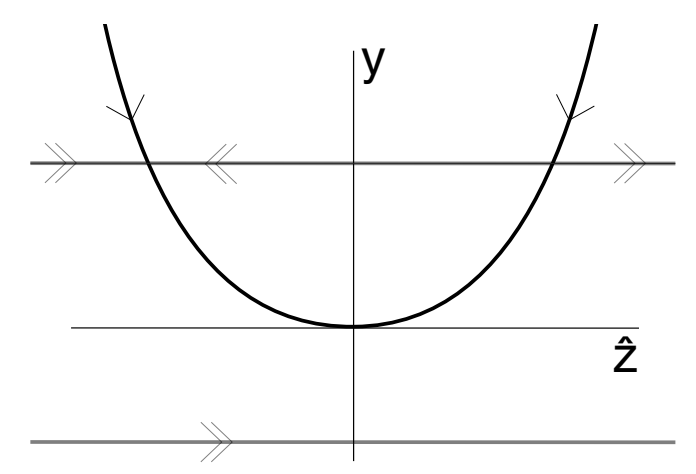

Figure 4: The dynamics of (2.21) with $x$ and $\eta$ both held constant.

Motivating the proof. We first consider the reduced system,

$$
\begin{aligned}
\dot{u} & =\frac{\sigma^{*}}{2} u-\hat{z} u \\
\dot{\hat{z}} & =\hat{z}^{2}-u,
\end{aligned}
$$

found by setting $x, \hat{y}, \eta$ and $\hat{\epsilon}$ all equal to zero in (2.19). This system describes the singular fast jump between $\mathcal{M}_{L, 0}$ and $\mathcal{M}_{R, 0}$, albeit only while this trajectory lies in a neighborhood of the local center manifold. The local dynamics about the origin are shown in Figure 5. The origin is a fixed point for this system and it has one unstable eigenvalue and one zero eigenvalue. The $\hat{z}$ axis is an invariant center manifold. Recall that the singular solution is comprised of the critical F-KPP front for $v_{1}=\beta$. The asymptotics of this front are given in (2.16) with $B<0$. Solutions departing the origin can be of two qualitative forms. There is a unique orbit departing in the one dimensional strong unstable manifold. All other trajectories depart asymptotic to the center manifold. Since $B<0$ for the critical F-KPP front, one readily observes that the singular solution falls into the latter case and this solution departs the origin asymptotic to the $\hat{z}$ axis. We note that if for some other front solution we have $B=0$ in (2.16), then the decay of the front is purely exponential and this solution lies in the strong unstable manifold of the origin. Note that the projective coordinates retain a quadratic approximation to the unstable manifold in $u_{1}, u_{2}$ coordinates and this explains why the eigenspace is not vertical.

Thus, $B<0$ in (2.16) is essential. Since this singular solution is asymptotic to the $\hat{z}$ axis, we get immediately that this trajectory intersects transversely with the unstable fiber associated to some $\hat{z}>0$ and sufficiently small. Furthermore, the invariance of the family of unstable fibers under the flow imply that this transverse intersection actually occurs for any $\hat{z}>0$. We may now explain our approach for the proof of Theorem 1. Fix $\delta>0$ so that the transverse intersection of the singular solution and unstable fiber associated to $\hat{z}=\delta$ occurs within the neighborhood $\Omega$. As a result of this transversality we can vary the $\eta$ parameter within some small neighborhood and retain a locally unique, transverse intersection of $W^{s}\left(P_{+}\right)$ and the unstable fiber at $\hat{z}=\delta$. Second, we can also vary the touch down point in $\mathcal{M}_{L, 0}$ in a neighborhood of $v_{1}=\beta$. This leads to another F-KPP equation, see (2.15), the asymptotics of which are again given by (2.16) with $B<0$, a continuous function of $v_{1}$. Again, we find a transverse intersection of the manifold $W^{s}\left(P_{+}\right)$with the unstable fiber at $\hat{z}=\delta$.

To complete the argument we need to track $W^{u u}\left(P_{-}\right)$as it evolves past this fold point. Theorem 2 provides the necessary machinery. For each $\eta$ and $\hat{\epsilon}>0$, the manifold $W^{u u}\left(P_{-}\right)$intersects the local center manifold transversely. The trajectory in this intersection can be mapped past the knee using the transition map $\pi$. We will show that the remainder of $W^{u u}\left(P_{-}\right)$gets mapped asymptotically close to the unstable fiber with basepoint $(x, \hat{y}, u, \hat{z}, \eta, \hat{\epsilon})^{T}=(x, 0,0, \delta, \eta, 0)^{T}$. 


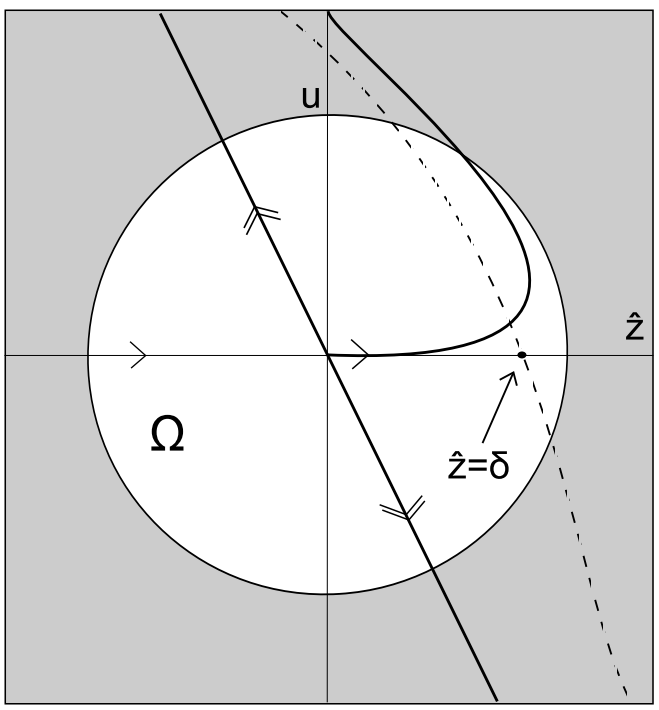

Figure 5: A schematic of the dynamics of (2.22). The singular orbit departs asymptotic to the $\hat{z}$ axis. The dashed line is the unstable fiber associated to $\hat{z}=\delta$ with $\delta$ chosen so that the intersection of this fiber with the singular orbit occurs within the neighborhood $\Omega$.

Setting up the sections to the flow. Define the transformation $\Psi\left(u_{1}, u_{2}, v_{1}, v_{2}, \sigma\right)$ as the composition of the transformations performed in section 2.3. Let,

$$
\mathcal{M}^{-}(\hat{\epsilon}):=\Psi\left(W^{u u}\left(P_{-}\right), \sigma\right),
$$

for the unstable manifold $W^{u u}\left(P_{-}\right)$and,

$$
\mathcal{M}^{+}(\hat{\epsilon}):=\Psi\left(W^{s}\left(P_{+}\right), \sigma\right),
$$

for the stable manifold of $P_{+}$. Note that these manifolds depend on $\epsilon$ or its rescaled form $\hat{\epsilon}$. We wish to compare these two manifolds in some section. Within the center manifold a natural choice is the section $\Delta^{\text {out }}$, defined in Theorem 2. Taking the union of unstable fibers corresponding to basepoints in the section $\Delta^{\text {out }}$ is also natural. At this point, we encounter a dimension problem as these objects lie in $\mathbb{R}^{6}$ while the manifolds $\mathcal{M}^{ \pm}(\hat{\epsilon})$ lie in $\mathbb{R}^{5}$. This extra dimension is the trivial direction in $\hat{\epsilon}$ so we resolve this with the introduction of the natural projection $\pi_{\hat{\epsilon}}:=(x, \hat{y}, u, \hat{z}, \eta, \hat{\epsilon}) \rightarrow(x, \hat{y}, u, \hat{z}, \eta)$. Then our choice of section is as follows,

$$
\Sigma^{\text {out }}:=\pi_{\hat{\epsilon}}\left(\bigcup_{\substack{p \in \Delta^{\text {out }} \\ \epsilon=0}} M_{u}(p)\right),
$$

where we recall that $M_{u}(p)$ is the unstable fiber associated to the basepoint $p$. The remainder of the proof amounts to finding descriptions of $\mathcal{M}^{ \pm}(\hat{\epsilon})$ in this section and showing that for each $\hat{\epsilon}$ sufficiently small there exists a unique orbit in the intersection of both manifolds. We select $(x, \hat{y}, \eta, c)$ coordinates for this section, where $(x, \hat{y}, \eta)$ parameterize the basepoint and $c$ gives the distance along the associated unstable fiber, see Lemma 2.2.

The manifold $\mathcal{M}^{-}(\hat{\epsilon})$ in $\Sigma^{\text {out }}$. We define the entry section $\Sigma^{\text {in }}$ in a similar manner to the exit section,

$$
\Sigma^{i n}:=\pi_{\hat{\epsilon}}\left(\bigcup_{\substack{p \in \Delta i n \\ \hat{\epsilon}=0}} M_{u}(p)\right) .
$$


This section effectively determines the boundary at which we transform from using the Fenichel description of the manifold $W^{u u}\left(P_{-}\right)$to using the center manifold geometry. The Fenichel description gives that for each $\hat{\epsilon} \geq 0$, the strong unstable manifold $\mathcal{M}^{-}(\hat{\epsilon})$ is transverse to $\Sigma^{i n}$ and its restriction to the center manifold has, for each $\eta$, a unique intersection with $\Delta^{i n}$.

We apply Theorem 2 to track the evolution of this basepoint as it evolves from $\Sigma^{i n}$ to $\Sigma^{\text {out }}$. We caution the reader that the Fenichel fibers and center manifold fibers are not necessarily equivalent (even after projection of the center manifold fibers into $\left.\mathbb{R}^{5}\right)$. In general, one would need to track each point in $\left(\mathcal{M}^{-}(\hat{\epsilon}), \hat{\epsilon}\right)$ separately; decomposing this manifold into a whole range of basepoints and corresponding points in the unstable fibers of these basepoints. Our task is simplified considerably by the exponential contraction of the transition map $\pi: \Delta^{i n} \rightarrow \Delta^{\text {out }}$ in Theorem 2. We track only the points in the intersection of $\mathcal{M}^{-}(\hat{\epsilon})$ and the section $\Delta^{i n}$. Denote these point $b^{i n}(\eta)$. At exit, the exponential contraction of the transition map implies that all other basepoints will be exponentially close to the basepoint $\pi\left(b^{i n}\right)$ at exit. The fibers depend smoothly on their basepoint so the unstable fibers will also be exponentially close to the unstable fiber $M_{u}\left(\pi\left(b^{i n}\right)\right)$.

All this is to say that we will really compare the manifold $\mathcal{M}^{+}(\hat{\epsilon})$ with the manifold,

$$
\mathcal{M}^{b}(\hat{\epsilon}):=\pi_{\hat{\epsilon}} \bigcup_{\tau \in \mathbb{R}^{+}} M_{u}\left(\phi\left(\tau, b^{i n}\right)\right),
$$

where we recall that $\phi$ is the flow of the full system. Our argument above implies that, near $\Sigma^{\text {out }}$ this manifold is $C^{1} \mathcal{O}\left(e^{-C / \hat{\epsilon}}\right)$ close to $\mathcal{M}^{-}(\hat{\epsilon})$. We have the following description of $\mathcal{M}^{b}(\hat{\epsilon})$ in the section $\Sigma^{\text {out }}$.

Lemma 2.3. For any $\delta>0$ and for $\hat{\epsilon}$ sufficiently small the strong unstable manifold $\mathcal{M}^{-}(\hat{\epsilon})$ is transverse to the section $\Sigma^{o u t}$, and the intersection of the manifold with this section is given as a graph over $c$ (the distance along the unstable fiber, see Lemma 2.2), $\eta$ and $\hat{\epsilon}$,

$$
\begin{aligned}
x(c, \eta, \hat{\epsilon}) & =\hat{\epsilon} \zeta_{1}(c, \eta, \hat{\epsilon}) \\
y(c, \eta, \hat{\epsilon}) & =-\Omega_{0} \hat{\epsilon}^{2 / 3}+\hat{\epsilon} \log (\hat{\epsilon}) \zeta_{2}(c, \eta, \hat{\epsilon}),
\end{aligned}
$$

with $\zeta_{i}$ smooth and $\left|\zeta_{i}\right|<C$, for some $C>0$ and independent of $\hat{\epsilon}$.

Proof: We will find a description of $\mathcal{M}^{b}(\hat{\epsilon})$ in the section $\Sigma^{\text {out }}$. We restrict our attention to a subset of this manifold near $\Sigma^{\text {out }}$. For any $\hat{\epsilon}>0$ we can write the orbit in the center manifold passing through $\Delta^{i n}$ at $b^{i n}$ as a graph $\hat{y}=\gamma(\hat{z})$. Restricting our attention to a neighborhood of $\hat{z}=\delta$, we find a $\kappa>0$ such that for $|\hat{z}-\delta|<\kappa$ we have

$$
\gamma(\hat{z})=-\Omega_{0} \hat{\epsilon}^{2 / 3}+\epsilon \log (\epsilon) \gamma_{1}(\epsilon, \hat{z})
$$

with $\gamma_{1}$ smooth and bounded independent of $\epsilon$. Let $p_{b}(\hat{z}, \eta, \hat{\epsilon}):=(0, \gamma(\hat{z}), 0, \hat{z}, \eta, \hat{\epsilon})^{T}$ be the basepoint of $\mathcal{M}^{b}(\hat{\epsilon})$. From Lemma 2.2, this manifold can be locally expressed as,

$$
\mathcal{M}^{b}(\hat{\epsilon})=\pi_{\hat{\epsilon}} \bigcup_{\substack{\hat{z} \in(\delta-\kappa, \delta+\kappa) \\ \eta \in \mathbb{R}, c_{b} \in \mathbb{R}}}\left(p_{b}+c_{b} \chi_{u}+J_{0}\left(p_{b}, c_{b}\right)+\hat{\epsilon} J_{1}\left(p_{b}, c_{b}\right)\right) .
$$

The section $\Sigma^{o u t}$ has a similar representation. Here we parameterize the basepoint by $p(x, \hat{y}, \eta)=(x, \hat{y}, 0, \delta, \eta, 0)^{T}$ and find,

$$
\Sigma^{\text {out }}=\pi_{\hat{\epsilon}} \bigcup_{\substack{p \in \Delta_{\text {out }} \\ c \in \mathbb{R}}}\left(p+c \chi_{u}+J_{0}(p, c)\right) .
$$

Let $\hat{c}=c_{b}-c$. Then the manifold $\mathcal{M}^{b}(\hat{\epsilon})$ intersects $\Sigma^{o u t}$ at those points where,

$$
\pi_{\hat{\epsilon}}\left(p_{b}-p+\hat{c} \chi_{u}+J_{0}\left(p_{b}, c_{b}\right)-J_{0}\left(p, c_{b}-\hat{c}\right)+\hat{\epsilon} J_{1}\left(p_{b}, c_{b}\right)\right)=0 .
$$

This is a system of five equations and seven variables. Note that the final equation for $\eta$ is trivial and so treating $\eta$ as parameter we reduce to a system of four equations and seven unknowns. Let $F^{-}\left(x, \hat{y}, \hat{c}, \hat{z}, c_{b}, \eta, \hat{\epsilon}\right)$ 
denote this set of four implicit equations. We have $F^{-}\left(0,0,0, \delta, c_{b}, \eta, 0\right)=0$ for all $c_{b}$ and $\eta$. Furthermore $D_{x, \hat{y}, \hat{c}, \hat{z}} F^{-}$is invertible for all $c_{b}$ and $\eta$ and for all $\hat{\epsilon}$ sufficiently small. An application of the Implicit Function Theorem gives $x, \hat{y}, \hat{c}, \hat{z}$ as a smooth function of $c_{b}, \eta$, and $\hat{\epsilon}$ for all $c_{b}$ and $\eta$ and for $\hat{\epsilon}$ sufficiently small. Using (2.20), we find,

$$
\begin{aligned}
x & =\epsilon \zeta_{1}\left(c_{b}, \eta, \hat{\epsilon}\right) \\
\hat{y} & =-\Omega_{0} \hat{\epsilon}^{2 / 3}+\hat{\epsilon} \log (\hat{\epsilon}) \zeta_{2}\left(c_{b}, \eta, \hat{\epsilon}\right) \\
c & =c_{b}-\hat{c}\left(c_{b}, \eta, \hat{\epsilon}\right) .
\end{aligned}
$$

The third equation can be inverted for all $c_{b}$, giving (2.23).

The manifold $\mathcal{M}^{+}(\hat{\epsilon})$ in $\Sigma^{\text {out }}$. We now consider $\mathcal{M}^{+}(\hat{\epsilon})$. We will show,

Lemma 2.4. There exists a constant $C_{k}(\delta)$, independent of $\hat{\epsilon}$ and a smooth function $h_{K P P}(\hat{y}, \eta)$ such that for all $|\hat{y}|<C_{k}$ and $|\eta|<C_{k}$ we have $\mathcal{M}^{+}(\hat{\epsilon}) \cap \Sigma^{\text {out }}$ is given by the graph

$$
\begin{aligned}
x(y, \eta, \hat{\epsilon}) & =-\frac{Q^{\prime}(\beta)}{a_{1}} \hat{y}+\frac{Q^{\prime}(\beta) \sigma^{*}}{2 a_{1}} \eta+\zeta_{3}(\hat{y}, \eta, \epsilon)+\epsilon \zeta_{3}(\hat{y}, \eta, \epsilon) \\
c(\hat{y}, \eta, \hat{\epsilon}) & =h_{K P P}(\hat{y}, \eta)+\epsilon \zeta_{4}(\hat{y}, \eta, \epsilon),
\end{aligned}
$$

where $\left|\zeta_{3}\right|<C\left(\hat{y}^{2}+\eta^{2}\right),\left|\zeta_{4}\right|<C,\left|\zeta_{5}\right|<C$ with $\zeta_{i}$ all smooth functions.

Proof: We track $W^{s}\left(P_{+}\right)$as it evolves backwards from $\mathcal{M}_{R, 0}$ to $\mathcal{M}_{L, 0}$. This evolution is given by the dynamics of the fast subsystem, (2.14). When $\hat{\epsilon}=0, v_{1}$ and $v_{2}$ are parameters, related by $H_{R}\left(v_{1}, v_{2}\right)=0$. We use $(2.17)$ to derive a local expression of this relationship in $(x, y)$ coordinates,

$$
x=-Q^{\prime}(\beta) \Gamma(\beta, r) y+\mathcal{O}\left(y^{2}\right) .
$$

Rescaling the $y$ component we find this relationship in terms of $\hat{y}$,

$$
x=-\frac{Q^{\prime}(\beta)}{a_{1}} \hat{y}+\frac{Q^{\prime}(\beta) \sigma^{*}}{2 a_{1}} \eta+\mathcal{O}\left(\hat{y}^{2}, \eta^{2}\right) .
$$

This equation gives the leading order expansion of the basepoints in $\Delta^{\text {out }}$ for which we expect an intersection of $\Sigma^{\text {out }}$ with $\mathcal{M}^{+}(\hat{\epsilon})$. We again use the coordinates $(x, \hat{y}, c, \eta)$ on the section $\Sigma^{\text {out }}$ and seek $c(x, \hat{y}, \eta)$ describing $\mathcal{M}^{+}(\hat{\epsilon})$ in this section.

Consider $\hat{\epsilon}=0$. As we discussed above, $B<0$ implies that the singular solution intersects transversely with the unstable fiber $M_{u}(0,0,0, \delta, 0,0)$. We track $W^{s}\left(P_{+}\right)$backwards along this solution and note that smoothness of both the solution with respect to initial conditions and the section $\Sigma^{\text {out }}$ with respect to its basepoint gives that this unique, transverse intersection persists locally for all $\hat{y}$ and $\eta$ in some small neighborhood of zero. We denote the $c$ value of this intersection by the smooth function $h_{K P P}(\hat{y}, \eta)$. Since $\mathcal{M}^{+}(\hat{\epsilon})$ depends smoothly on $\hat{\epsilon}$ and $\Sigma^{\text {out }}$ is independent of $\hat{\epsilon}$, we have that this intersection persists for small positive $\hat{\epsilon}$. This establishes $(2.24)$.

Lemma 2.5. For $\epsilon$ sufficiently small, $\mathcal{M}^{-}(\hat{\epsilon})$ and $\mathcal{M}^{+}(\hat{\epsilon})$ have a locally unique intersection with wavespeed given by,

$$
\eta(\epsilon)=-\frac{\Omega_{0} a_{1}^{2 / 3} \Gamma(\beta, r)^{2 / 3}}{\sqrt{1-a_{1} \beta}} \epsilon^{2 / 3}+\mathcal{O}(\epsilon \log (\epsilon)) .
$$


Proof: An intersection of these manifolds corresponds to a solution of the implicit equations (2.23) and (2.24). The existence and uniqueness of such a solution is a consequence of the Implicit Function Theorem. Consider the map $F(x, \hat{y}, \eta, c, \hat{\epsilon}): \mathbb{R}^{4} \times \mathbb{R} \rightarrow \mathbb{R}^{4}$ given by equations (2.23) and (2.24). Writing out the Newton iteration, $F$ has a zero solution exactly when the following map has a fixed point

$$
G(x, \hat{y}, \eta, c, \hat{\epsilon}):=(x, \hat{y}, \eta, c)-\left[D_{x, \hat{y}, \eta, c} F\left(0,0,0, h_{K P P}(0,0), 0\right)\right]^{-1} F(x, \hat{y}, \eta, c, \hat{\epsilon}) .
$$

The Jacobian,

$$
D_{x, y, u, \eta} F\left(0,0,0, h_{K P P}(0,0), 0\right)=\left(\begin{array}{cccc}
1 & 0 & 0 & 0 \\
0 & 1 & 0 & 0 \\
1 & \frac{Q^{\prime}(\beta)}{a_{1}} & -\frac{Q^{\prime}(\beta) \sigma^{*}}{2 a_{1}} & 0 \\
0 & \frac{\partial h_{K P P}}{\partial \hat{y}}(0,0) & \frac{\partial h_{K P P}}{\partial \eta}(0,0) & 1
\end{array}\right)
$$

is invertible provided $Q^{\prime}(\beta) \neq 0$, which follows from its definition and guarantees that the manifolds intersect transversely. The Implicit Function Theorem then provides a solution $x(\epsilon), y(\epsilon), u(\epsilon), \eta(\epsilon)$ that is $\mathcal{O}\left(\epsilon^{2 / 3}\right)$ close to the singular solution. Since we desire an explicit leading order approximation, we write out the map $G$,

$$
G(x, y, u, \eta)=\left(\begin{array}{c}
\epsilon \zeta_{1}(c, \eta, \hat{\epsilon}) \\
-\Omega_{0} \hat{\epsilon}^{2 / 3}+\hat{\epsilon} \log (\hat{\epsilon}) \zeta_{2}(c, \eta, \hat{\epsilon}) \\
-\frac{2}{\sigma^{*}} \Omega_{0} \hat{\epsilon}^{-2 / 3}-\frac{2 a_{1}}{Q^{\prime}(\beta) \sigma^{*}} \zeta_{3}(\hat{y}, \eta, \hat{\epsilon})+\hat{\epsilon} \log (\hat{\epsilon}) \zeta_{6}(x, \hat{y}, \eta, c, \hat{\epsilon}) \\
h_{K P P}(\hat{y}, \eta)+\frac{\partial h_{K P P}}{\partial \hat{y}}(0,0)\left(\hat{y}+\Omega_{0} \hat{\epsilon}^{2 / 3}\right)-\frac{\partial h_{K P P}}{\partial \eta}(0,0)\left(\eta-\frac{2 \Omega_{0}}{\sigma^{*}} \hat{\epsilon}^{2 / 3}\right)+\zeta_{7}(x, \hat{y}, \eta, c, \hat{\epsilon})
\end{array}\right) .
$$

Here $\left|\zeta_{6}\right|<C$ and $\left|\zeta_{7}\right|<C\left(|\hat{\epsilon}|+\hat{y}^{2}+\eta^{2}\right)$. The fixed point equation then implies that $x(\hat{\epsilon})=\mathcal{O}(\hat{\epsilon})$ and $\hat{y}(\hat{\epsilon})=\mathcal{O}\left(\hat{\epsilon}^{2 / 3}\right)$. In turn, we have that the $\zeta_{3}$ term in the third row is higher order and we conclude,

$$
\eta=-\frac{\Omega_{0}}{\sqrt{1-a_{1} \beta}} \hat{\epsilon}^{2 / 3}+\mathcal{O}(\hat{\epsilon} \log (\epsilon)) .
$$

After the rescaling, $\hat{\epsilon}=a_{1} \Gamma(\beta, r) \epsilon$, we arrive at the result.

This establishes the main claims of Theorem 1. To conclude the proof, it remains to verify the positivity and monotonicity of this front.

Lemma 2.6. The traveling front constructed in Lemma 2.5 is everywhere positive and monotone.

Proof: Positivity is straightforward. While in the neighborhood of the jump point in $\mathcal{M}_{L, \epsilon}$, the only way to achieve negative values of $u_{1}$ is to reach $\hat{z}=-\infty$ in finite time, which is not possible. When exiting $\Omega$, we transform to the Fenichel geometry, from which a simple eigenvector calculation gives positivity of the solution for sufficiently small $\epsilon$. A similar argument gives positivity of the $v$ component near $P_{+}$.

We now consider monotonicity. Considering $v$ first, we see from the functional form of $\mathcal{M}_{L, 0}$ and $\mathcal{M}_{R, 0}$ that $v_{2}$ is bounded away from zero except near the two fixed points $P_{+}$and $P_{-}$. These fixed points do not vary with $\epsilon$, and their stable and unstable manifolds are locally tangent to the stable and unstable eigenvectors computed in (2.6) and (2.8). The dynamics near $P_{ \pm}$are dominated by the weaker eigendirections, both of which satisfy $v_{2}<0$.

Now consider $u_{2}$, which we will show is always non-zero. We note that $u_{2}$ is nearly zero while the orbit is near the slow manifold and is positive during the fast transition between slow manifolds. We argue in pieces. First consider the solution near $\mathcal{M}_{L, \epsilon}$. Suppose that the $u$ component is not monotone. Since $u$ must be positive this implies the existence of at least two critical points, one a local maximum and one a local 
minimum (alternatively there could be one critical point with zero second derivative, but a similar argument will work in this case). Consider the traveling wave equation,

$$
\epsilon^{2} u_{\xi \xi}-\epsilon \sigma u_{\xi}+\left(1-u-a_{1} v\right) u=0 .
$$

Near $\mathcal{M}_{L, \epsilon}$, we find that the nonlinearity must be positive, so we find a contradiction at the local minimum where the second derivative is also positive. Now as we noted above, $u_{2}>0$ during the transition between $\mathcal{M}_{L, \epsilon}$ and $\mathcal{M}_{R, \epsilon}$. Upon entry into $\mathcal{M}_{R, \epsilon}$, we can use the Fenichel geometry to exclude the possibility of $u_{2}$ reaching zero. Indeed, the fast stable eigenspace for $\mathcal{M}_{R, \epsilon}$ is $\mathcal{O}(\epsilon)$ close to $\left(1, \mu_{\text {fast }}, 0,0\right)^{T}$ with $\mu_{\text {fast }}<0$. Along $\mathcal{M}_{R, \epsilon}$, we have $u_{1}=1-a_{1} v_{1}+\mathcal{O}(\epsilon)$. Differentiating we find $\epsilon^{-1} u_{2}=-a_{1} v_{2}+\mathcal{O}(\epsilon)$. Then for sufficiently small $\epsilon$ and $v_{2}$ bounded away from zero we get that $u_{2} \neq 0$ along $\mathcal{M}_{L, \epsilon}$. It remains to show that $u$ is monotone in a neighborhood of $P_{+}$. This follows from considering the eigenvectors in (2.6), where a non-zero $u$ component in $n_{2}^{-}$implies the monotonicity of $u$ in a neighborhood of $P_{+}$.

\section{Stability Analysis}

We now turn to the asymptotic stability of the critical front constructed in Theorem 1. This result will justify our use of the term pushed front. The stability of monotone traveling fronts for equations of the form (1.1) has been studied in [21] under the assumption that the homogeneous steady states $P_{+}$and $P_{-}$ are stable. We adopt this line of analysis to the present case. The primary obstacle to the application of the methods in [21] is the presence of essential spectrum in the right half plane. We work in a weighted Banach space that eliminates this problem.

Let $U(\xi)$ and $V(\xi)$ denote the traveling wave from Theorem 1. Let $u(t, \xi)=U(\xi)+p(t, \xi)$ and $v(t, \xi)=$ $V(\xi)+q(t, \xi)$ and linearize to find

$$
\begin{aligned}
p_{t} & =\epsilon^{2} p_{\xi \xi}-\epsilon \sigma p_{\xi}+\left(1-a_{1} V(\xi)-2 U(\xi)\right) p-a_{1} U q \\
q_{t} & =q_{\xi \xi}-\epsilon \sigma q_{\xi}-r a_{2} V(\xi) p+r\left(1-a_{2} U(\xi)-2 V(\xi)\right) q .
\end{aligned}
$$

Let $\mathcal{L}$ denote the right hand side of the previous vector equation. Then $\mathcal{L}: C_{\text {unif }}(\mathbb{R}) \times C_{\text {unif }}(\mathbb{R}) \rightarrow$ $C_{\text {unif }}(\mathbb{R}) \times C_{\text {unif }}(\mathbb{R})$. Let $W(\xi)$ be the weight introduced in $(2.3)$. We will consider the stability of the traveling front in the weighted space $C_{\text {unif,W }}(\mathbb{R}):=\left\{u \in C_{\text {unif }}(\mathbb{R}) \mid u(t, \cdot) W(\cdot) \in C_{\text {unif }}(\mathbb{R})\right\}$. To do this, we consider the operator $\mathcal{L}_{W}: C_{\text {unif }}(\mathbb{R}) \times C_{\text {unif }}(\mathbb{R}) \rightarrow C_{\text {unif }}(\mathbb{R}) \times C_{\text {unif }}(\mathbb{R})$ given by

$$
\mathcal{L}_{W}=\left(\begin{array}{cc}
W(\xi) \epsilon^{2} \partial_{\xi \xi} \frac{1}{W(\xi)}-\epsilon \sigma W(\xi) \partial_{\xi} \frac{1}{W(\xi)}+\left(1-a_{1} V(\xi)-2 U(\xi)\right) & -a_{1} W(\xi) U(\xi) \\
-r a_{2} \frac{V(\xi)}{W(\xi)} & \partial_{\xi \xi}-\epsilon \sigma \partial_{\xi}+r\left(1-a_{2} U(\xi)-2 V(\xi)\right)
\end{array}\right)
$$

Since $W(\xi)^{-1}$ is bounded and $U(\xi) \in C_{\text {unif,W }}(\mathbb{R})$ we have that $\mathcal{L}_{W}$ is a well defined operator from $C_{\text {unif }}(\mathbb{R}) \times$ $C_{\text {unif }}(\mathbb{R}) \rightarrow C_{\text {unif }}(\mathbb{R}) \times C_{\text {unif }}(\mathbb{R})$. We will establish the following result.

Theorem 3. The spectrum of $\mathcal{L}_{W}$ lies entirely in the left half plane, with the exception of a simple eigenvalue at the origin due to translational invariance.

We will proceed as follows. We refer the reader to [27] for a review of spectral stability of traveling fronts. The spectrum of $\mathcal{L}_{W}$ consists of those values of $\lambda \in \mathbb{C}$ for which $\mathcal{L}_{W}-\lambda$ is not invertible. The spectrum can be decomposed into two disjoint sets: the essential spectrum and the point spectrum. The essential spectrum are those values of $\lambda$ for which the linear operator $\mathcal{L}_{W}-\lambda$ is not Fredholm index zero. The point spectrum consists of isolated eigenvalues where the operator is Fredholm index zero but has a non-trivial kernel. 


\subsection{The essential spectrum}

The linearized eigenvalue problem $\mathcal{L}_{W}-\lambda=0$ is a system of second order ordinary differential equations. As such, it can be expanded into a system of first order ordinary differential equations,

$$
\phi_{\xi}=A(\xi, \lambda) \phi .
$$

The boundary of the essential spectrum consists of those values of $\lambda$ for which the limiting matrix $A_{ \pm}(\lambda):=$ $\lim _{\xi \rightarrow \pm \infty} A(\xi, \lambda)$ has purely imaginary eigenvalues. Note that as $\xi \rightarrow \pm \infty$ the operator $\mathcal{L}_{W}$ converges to a triangular operator and so the eigenvalues of $A_{ \pm}$are simply the eigenvalues of the diagonal elements. Thus, the boundary of the essential spectrum consists of the following four curves,

$$
\begin{aligned}
& S_{p}^{-}:=\left\{-\epsilon^{2} k^{2}-\epsilon \sigma i k-4 \epsilon \sqrt{1-a_{1}} i k-a_{1}(1-\beta) \quad \mid k \in \mathbb{R}\right\} \\
& S_{q}^{-}:=\left\{-k^{2}-\epsilon \sigma i k-r \quad \mid \quad k \in \mathbb{R}\right\} \\
& S_{p}^{+}:=\left\{-\epsilon^{2} k^{2}-\epsilon \sigma i k-1 \quad \mid k \in \mathbb{R}\right\} \\
& S_{q}^{+}=\left\{-k^{2}-\epsilon \sigma i k-r\left(a_{2}-1\right) \quad \mid k \in \mathbb{R}\right\}
\end{aligned}
$$

Note that for all parameters under consideration the essential spectrum of $\mathcal{L}_{W}$ lies in the left half-plane.

\subsection{The point spectrum}

It remains to exclude the possibility of the point spectrum of $\mathcal{L}_{W}$ in the right half plane. Our proof will rely on the monotonicity of the front and the following comparison principle. We define the ordering

$$
\left(u_{1}, v_{1}\right) \succeq\left(u_{2}, v_{2}\right) \Longleftrightarrow u_{1}(\xi) \geq u_{2}(\xi) \text { and } v_{1}(\xi) \leq v_{2}(\xi) \text { for all } \xi \in \mathbb{R} .
$$

We have the following facts,

- $\mathcal{L}_{W}$ is sectorial and generates an analytic semigroup which is positive with respect to this comparison principle. Due to the location of the essential spectrum, the only possible spectra in the closed right half plane is point spectrum.

- The point spectrum is real since the semigroup is positive.

The positivity of the semigroup implies that the point spectrum is additive cyclic, $\left(\lambda=\lambda_{r}+i \lambda_{i} \in \sigma\left(\mathcal{L}_{W}\right)\right.$ implies $\lambda_{r}+i z \lambda_{i} \in \sigma\left(\mathcal{L}_{W}\right)$ for all $z \in \mathbb{Z}$, see [9]). Since the spectrum lies in a sector, we conclude that it must be real.

- The kernel of $\mathcal{L}_{W}$ is one-dimensional and spanned by $U_{0}(\xi)=\left(\partial_{\xi} U(\xi), \partial_{\xi} V(\xi)\right) \succeq 0$.

This follows from the transversality of $W^{u u}\left(P_{-}\right)$and $W^{s}\left(P_{+}\right)$in Theorem 1.

- The adjoint problem $\mathcal{L}_{W}^{*}$ has a non-trivial, one dimensional kernel spanned by $P^{*}=\left(p^{*}, q^{*}\right)$ with $\left(p^{*}, q^{*}\right) \succeq(0,0)$ (see Lemma A.2 of [22]).

$\mathcal{L}_{W}$ is Fredholm index zero which implies that $\mathcal{L}_{W}^{*}$ has a one dimensional kernel. That this kernel lies in the positive cone can be proved by contradiction using the same techniques as [22].

Therefore, to prove spectral stability we must rule out the possibility of unstable point spectrum for real, non-negative values of $\lambda$. To begin, suppose the existence of an eigenvalue for some positive value of $\lambda$. We will argue by contradiction. Denote the corresponding eigenfunction $P=(p, q)$. It is easy to see that $P(\xi)$ is not identically zero in either of its components. Fix a maximal interval $\Delta^{p}$ for which $p(\xi)>0$. We claim that there exists a value $\xi_{2} \in \Delta^{p}$ for which $q(\xi)<0$. Suppose this were not the case, then

$$
\begin{aligned}
0 & =\int_{\Delta^{p}}\left(\left(\mathcal{L}_{W}-\lambda\right) P\right)_{1} p^{*}(\xi) d \xi=\left[W(\xi) p^{*}(\xi) \partial_{\xi} \frac{p(\xi)}{W(\xi)}\right]_{\partial \Delta^{p}} \\
& -\int_{\Delta^{p}}\left(a_{1} W(\xi) U(\xi) q(\xi) p^{*}(\xi)-r a_{2} \frac{V(\xi)}{W(\xi)} p(\xi) q^{*}(\xi)+\lambda p(\xi) p^{*}(\xi)\right) d \xi<0 .
\end{aligned}
$$


This implies the existence of a non-trivial, maximal interval $\Delta^{q}=\left(\xi_{-}^{p}, \xi_{+}^{p}\right)$ for which $q(\xi)<0$. Let $\Delta=$ $\Delta^{p} \bigcup \Delta^{q}$. A similar argument gives that $p(\xi) \leq 0$ on $\Delta \backslash \Delta^{p}$ and $q(\xi) \geq 0$ on $\Delta \backslash \Delta^{q}$. Define $\tilde{P}=(\tilde{p}, \tilde{q})$ by $\tilde{p}(\xi)=p(\xi)$ for all $\xi \in \Delta^{p}$ and zero otherwise. $\tilde{q}$ is defined analogously. Since $\tilde{P} \succeq(0,0)$ we have in a distributional sense that

$$
\int_{\mathbb{R}}\left(\left(\mathcal{L}_{W}-\lambda\right) \tilde{P}, P^{*}\right) d \xi=-\lambda \int_{\mathbb{R}}\left(\tilde{P}, P^{*}\right) d \xi \leq 0 .
$$

On the other hand,

$$
\left(\mathcal{L}_{W}^{11}-\lambda\right) \tilde{p}+\mathcal{L}_{W}^{12} \tilde{q}=\left\{\begin{array}{cc}
-a_{1} W(\xi) U(\xi) \tilde{q} & \xi \in \Delta^{q} \backslash \Delta^{p} \\
-a_{1} W(\xi) U(\xi)(\tilde{q}-q) & \xi \in \Delta^{p} \\
\epsilon^{2} p_{\xi}\left(\xi_{-}^{p}\right) \delta\left(\xi-\xi_{-}^{p}\right) & \xi=\xi_{-}^{p} \\
-\epsilon^{2} p_{\xi}\left(\xi_{+}^{p}\right) \delta\left(\xi-\xi_{+}^{p}\right) & \xi=\xi_{+}^{p} \\
0 & \text { otherwise }
\end{array}\right.
$$

again in a distributional sense.

From this,

$$
\int_{\mathbb{R}}\left(\left(\mathcal{L}_{W}^{11}-\lambda\right) \tilde{p}+\mathcal{L}_{W}^{12} \tilde{q}\right) p^{*}(\xi) d \xi \geq 0
$$

An analogous result holds for

$$
\int_{\mathbb{R}}\left(\mathcal{L}_{W}^{21} \tilde{p}+\left(\mathcal{L}_{W}^{22}-\lambda\right) \tilde{q}\right) q^{*}(\xi) d \xi \geq 0,
$$

which combined with (3.3) implies that $\lambda$ can not be positive.

It remains to show that $\lambda=0$ is a simple eigenvalue. Since we have that the geometric multiplicity of zero is one, we must simply eliminate the possibility of a higher algebraic multiplicity. Suppose now that there exists a non-zero function $R$ such that $\mathcal{L}_{W} R=U_{0}$. Taking the inner product,

$$
\int_{\mathbb{R}}\left(U_{0}, P^{*}\right) d \xi=\int_{\mathbb{R}}\left(\mathcal{L}_{W} R, P^{*}\right) d \xi=\int_{\mathbb{R}}\left(R, \mathcal{L}_{W}^{*} P^{*}\right) d \xi=0
$$

we find a contradiction since the first integral is necessarily non-zero due to the fact that $\left(U_{0}, P^{*}\right)$ is everywhere positive.

\subsection{Nonlinear Stability}

We now consider the nonlinear stability of the front solution in this weighted Banach Space. As we did for the linearization, let $u(t, \xi)=U(t, \xi)+W(\xi) p(t, \xi)$ and $v(t, \xi)=V(\xi)+q(t, \xi)$ and determine the full nonlinear stability problem,

$$
\left(\begin{array}{c}
p_{t} \\
q_{t}
\end{array}\right)=\mathcal{L}_{W}\left(\begin{array}{c}
p \\
q
\end{array}\right)+\mathcal{N}_{W}(\xi, p, q)
$$

for $(p, q) \in C_{\text {unif }}(\mathbb{R}) \times C_{\text {unif }}(\mathbb{R})$ with

$$
\mathcal{N}_{W}(\xi, p, q)=\left(\begin{array}{c}
-\left(\frac{p}{W}+a_{1} q\right) p \\
-r\left(a_{2} \frac{p}{W}+q\right) q
\end{array}\right)
$$

Again, since $W(\xi)^{-1}$ is bounded, the nonlinear problem is well-posed and the nonlinearity is locally Lipschitz.

Theorem 4. Fix $\epsilon>0$. Let $\phi(t, u, v)$ be the flow associated to (1.1). Suppose that,

$$
\left\|\left(U_{0}(\cdot), V_{0}(\cdot)\right)-(U, V)\right\|_{C_{u n i f, W}(\mathbb{R}) \times C_{u n i f}(\mathbb{R})}<\delta,
$$

for some $\delta$ sufficiently small. Then there exists $C>0, \kappa>0$ and $\tau$ so that

$$
\left\|\phi\left(t, U_{0}, V_{0}\right)-(U(x-\epsilon \sigma t+\tau), V(x-\epsilon \sigma t+\tau))\right\|_{C_{u n i f, W}(\mathbb{R}) \times C_{u n i f}(\mathbb{R})} \leq C e^{-\kappa t} .
$$


The operator $\mathcal{L}_{W}$ is sectorial with an isolated eigenvalue of multiplicity one at the origin. The proof is a standard application of results in [15].

Remark 2. We note that the existence of a comparison principle for (1.1) can lead to a stronger result than that of Theorem 4. For example, the authors in [26] show that the selected front for this system is always the slowest monotone front. Here, we emphasize the marginal stability criterion, as this criterion applies to a larger set of examples, see the discussion at the end of the paper.

In order to appeal to the results of [26], we must show that the front we constructed in Theorem 1 is the slowest monotone front. We sketch the argument. Assume there exists a monotone front, traveling with speed $\sigma<\sigma_{\text {crit }}$. The decay rates of the u component of the front can be found by considering (2.5) and (2.7). Near $P_{-}$, this decay is given by $\mu_{1}^{+}\left(\sigma_{\text {crit }}\right)$. For any slower front, this decay is given by one of $\mu_{1}^{+}(\sigma)$ or $\mu_{1}^{-}(\sigma)\left(\mu_{2}^{+}(\sigma)\right.$ can be excluded because any front approaching along the corresponding eigenvector is necessarily non-monotone). Either way, one can calculate that the decay is weaker for the slower front. Near $P_{+}$a similar estimate holds, showing that the decay of the $u$ component is stronger for slower fronts. Therefore, there exists a translate of the slower front, $U_{\sigma}$ such that $U_{\sigma}>U$ for all $\xi$. An analogous result holds for the $v$ component and it is possible to find a translate of the slower front so that both $U_{\sigma}>U$ and $V_{\sigma}<V$ for all $\xi \in \mathbb{R}$. These fronts propagate to the left and therefore we have a contradiction as the faster front will eventually catch up to and pass the slower front, violating the comparison principle.

\section{Linear versus nonlinear spreading speeds}

In this final section, we discuss the linear spreading speed for system (1.1) and its relation to the nonlinear front from Theorem 1. We begin with a general discussion of linear spreading speeds and their derivation using the pointwise Green's function. We then use this framework to calculate the linear spreading speed for perturbations of $P_{-}$. The linear spreading speed for this system is found to be much faster than the spreading speed of the nonlinear front constructed above. We conclude with an explanation of why this fast spreading does not pose an obstacle to stability of the slower front.

\subsection{The Linear Spreading Speed}

For any value of $s>0$,

$$
u(t, x+s t)=0, \quad v(t, x+s t)=1,
$$

is a nonlinear solution to the partial differential equation (1.1). It is clear that this solution is unstable for all values of $s$. Therefore, small, compactly supported perturbations of this solution will grow in norm and spread spatially. In this section, we will discuss the spreading speed of such perturbations. We refer to $[2,19,29]$ for information on linear spreading speeds in general systems. Particular examples where the linear spreading speed has been calculated for non-scalar reaction-diffusion equations can be found in $[3,10,13]$. Note that we elect to work with a wavespeed $s$ instead of $\epsilon \sigma$ as we did before.

We linearize the partial differential equation (1.1) about the homogeneous steady state $P_{-}$, and view the evolution in a moving frame, with $\xi=x+s t$. The linearized equations in this frame are,

$$
\begin{aligned}
p_{t} & =\epsilon^{2} p_{\xi \xi}-s p_{\xi}+\left(1-a_{1}\right) p \\
q_{t} & =q_{\xi \xi}-s q_{\xi}-r q-r a_{2} p .
\end{aligned}
$$

We assume that the initial perturbation is compactly supported. We wish to determine whether this perturbation grows or decays within some finite window of observation. This information will allow us to compute the linear spreading speed as the supremum over $s$ for which exponential growth is observed in this finite window. This speed can be calculated in several ways. We will explain one approach, making use of the Laplace transform and pointwise Green's function. We will outline this approach in general and then apply it to the particular case of equations (4.1). To understand our emphasis on the pointwise Green's function, 
we note that we are particularly interested in differentiating between two different types of spreading: one due to poles of the Green's function and another that arises due to branch poles.

Denote the right hand side of $(4.1)$ by $\mathcal{L}_{-}(\xi)$. After a Laplace transform in time, the linearized partial differential equation is reduced to a system of second order inhomogeneous ordinary differential equations,

$$
\left(\mathcal{L}_{-}-\lambda\right)\left(\begin{array}{c}
p(\xi, \lambda) \\
q(\xi, \lambda)
\end{array}\right)=-\left(\begin{array}{c}
p_{0}(\xi) \\
q_{0}(\xi)
\end{array}\right)
$$

This system can be solved via the pointwise Green's function $G_{\lambda}(\xi-y)$. We remark that to the right of the curve $\lambda=-\epsilon^{2} k^{2}+s i k+\left(1-a_{1}\right), G_{\lambda}$ is related to the resolvent via $\left(\mathcal{L}_{-}-\lambda\right)^{-1} \phi=\int_{\mathbb{R}} G_{\lambda}(\xi-y) \phi(y) d y$. Since the resolvent is analytic on this domain so is $G_{\lambda}$. The advantage of $G_{\lambda}$ over the resolvent operator is that the Green's function can naturally be continued into the essential spectrum while a similar continuation of the resolvent requires a restriction to a smaller function space.

Recall that the Green's function is constructed as a linear combination of all bounded solutions on either half-line with the requirement that $G_{\lambda}$ be continuous at $\xi=y$ with a jump discontinuity of prescribed size in its derivative. To find all bounded solutions, the second order equation $\left(\mathcal{L}_{-}-\lambda\right) \phi=0$ can be transformed into a system of first order equations in the usual manner. In this case, the system is constant coefficient and the Green's function can be compiled from the fundamental solution of (4.1) and the stable and unstable projections. To the right of the essential spectrum this is all straightforward.

Since $G_{\lambda}$ is defined pointwise, extending it into the essential spectrum only requires that one can analytically continue these stable and unstable subspaces. This gives rise to two possible mechanisms by which singularities of $G_{\lambda}$ can be created. First, for some value of $\lambda$ the eigenvalues lose analyticity so that no analytic continuation of $G_{\lambda}$ is possible. The second possibility is that for some value of $\lambda$ the eigenvalues remain analytic, but the extended stable and unstable subspaces do not span the whole space. If this is the case, it is unlikely that the jump discontinuity condition for $G_{\lambda}$ at $\xi=y$ can be met. This gives rise to a pole singularity of the Green's function. In the latter case, one has a meromorphic extension of the Green's function and we are free to continue this extension further into the complex plane.

The time dependent Green's function can then be determined by inverse Laplace Transform,

$$
G(\xi, t, y)=\frac{1}{2 \pi i} \int_{\Gamma} e^{\lambda t} G_{\lambda}(\xi-y) d \lambda .
$$

The contour $\Gamma$ must be placed to the right of all singularities of $G_{\lambda}$. If $G_{\lambda}$ can be analytically continued to the whole right half plane then by Cauchy's Theorem the contour $\Gamma$ can be placed fully in the stable half plane and pointwise decay is achieved.

We have thusfar ignored the role of the wavespeed $s$. The singularities of $G_{\lambda}$ depend continuously on $s$ and therefore by altering the value of $s$ we can change where these singularities lie. The linear spreading speed is found as the largest value of $s$ for which all singularities lie on or to the left of the imaginary axis.

\subsection{The explicit Green's function}

The system (4.1) is just simple enough, meaning triangular with second order operators on the diagonal, that we may compute the Green's function directly. Of critical importance are the generalized eigenvalues of the operators on the diagonal, which we denote $\mathcal{L}_{1}-\lambda$ and $\mathcal{L}_{2}-\lambda$, with definitions,

$$
\mathcal{L}_{1}=\epsilon^{2} \partial_{\xi}^{2}-s \partial_{\xi}+\left(1-a_{1}\right), \quad \mathcal{L}_{2}=\partial_{\xi}^{2}-s \partial_{\xi}-r .
$$

Their eigenvalues are roots of the dispersion relation, which relates spatial modes, $e^{\nu \xi}$, to temporal growth rates, $e^{\lambda t}$. The dispersion relation can be derived using the ansatz $p(t, x)=p_{0} e^{\lambda t+\nu x}$ and $q(t, x)=q_{0} e^{\lambda t+\nu x}$. In a co-moving frame $\xi=x+s t$, the dispersion relation is

$$
d(\lambda, \nu):=d_{1}(\lambda, \nu) d_{2}(\lambda, \nu)=\left(\epsilon^{2} \nu^{2}-s \nu+\left(1-a_{1}\right)-\lambda\right)\left(\nu^{2}-s \nu-r-\lambda\right) .
$$


The roots of the dispersion relation are computed explicitly,

$$
\begin{aligned}
\nu_{1}^{ \pm}(\lambda, s) & =\frac{s}{2 \epsilon^{2}} \pm \frac{1}{2 \epsilon^{2}} \sqrt{s^{2}-4 \epsilon^{2}\left(1-a_{1}\right)+4 \epsilon^{2} \lambda} \\
\nu_{2}^{ \pm}(\lambda, s) & =\frac{s}{2} \pm \frac{1}{2} \sqrt{s^{2}+4 r+4 \lambda} .
\end{aligned}
$$

The pointwise Green's function must satisfy,

$$
\left(\begin{array}{cc}
\mathcal{L}_{1}-\lambda & 0 \\
-r a_{2} & \mathcal{L}_{2}-\lambda
\end{array}\right)\left(\begin{array}{cc}
G_{\lambda}^{11} & G_{\lambda}^{12} \\
G_{\lambda}^{21} & G_{\lambda}^{22}
\end{array}\right)=\left(\begin{array}{cc}
\delta(y) & 0 \\
0 & \delta(y)
\end{array}\right)
$$

Calculating $G_{\lambda}^{11}$ first, we find

$$
G_{\lambda}^{11}(\xi-y)=\left\{\begin{array}{lll}
\frac{1}{\nu_{1}^{-}-\nu_{1}^{+}} e^{\nu_{1}^{-}(\lambda)(\xi-y)} & \text { for } \quad \xi>y \\
\frac{1}{\nu_{1}^{-}-\nu_{1}^{+}} e^{\nu_{1}^{+}(\lambda)(\xi-y)} & \text { for } \quad \xi<y
\end{array}\right.
$$

It is easy to see that $G_{\lambda}^{12}=0$ and that $G_{\lambda}^{22}$ satisfies a similar equation to (4.3). Determining $G_{\lambda}^{21}$ requires solving the equation,

$$
\left(\mathcal{L}_{2}-\lambda\right) G^{21}=r a_{2} G^{11} .
$$

Recall that to the right of the essential spectrum, $G_{\lambda}^{11}$ is bounded and the operator $\mathcal{L}_{2}-\lambda$ is invertible so an analytic solution $G_{\lambda}^{21}$ necessarily exists on this subset of the complex plane. We find,

$$
G_{\lambda}^{21}(\xi-y)=\left\{\begin{array}{ll}
c_{1}(\lambda) e^{\nu_{2}^{-}(\lambda)(\xi-y)}+\frac{r a_{2}}{\nu_{1}^{-}-\nu_{1}^{+}} \frac{1}{d_{v}\left(\nu_{1}^{-}, \lambda\right)} e^{\nu_{1}^{-}(\lambda)(\xi-y)} & \text { for } \quad \xi>y \\
c_{2}(\lambda) e^{\nu_{2}^{+}(\lambda)(\xi-y)}+\frac{r a_{2}}{\nu_{1}^{-}-\nu_{1}^{+}} \frac{1}{d_{v}\left(\nu_{1}^{+}, \lambda\right)} e^{\nu_{1}^{+}(\lambda)(\xi-y)} & \text { for } \quad \xi<y
\end{array},\right.
$$

where $c_{1}(\lambda)$ and $c_{2}(\lambda)$ are constants determined by continuity and differentiability requirements on $G_{\lambda}^{21}$. These constants are

$$
\begin{aligned}
& c_{1}(\lambda)=\frac{r a_{2}}{\left(\nu_{1}^{-}-\nu_{1}^{+}\right)\left(\nu_{2}^{-}-\nu_{2}^{+}\right)}\left(\frac{\nu_{1}^{+}-\nu_{2}^{+}}{d_{2}\left(\nu_{1}^{+}, \lambda\right)}-\frac{\nu_{1}^{-}-\nu_{2}^{+}}{d_{2}\left(\nu_{1}^{-}, \lambda\right)}\right) \\
& c_{2}(\lambda)=\frac{r a_{2}}{\left(\nu_{1}^{-}-\nu_{1}^{+}\right)\left(\nu_{2}^{-}-\nu_{2}^{+}\right)}\left(\frac{\nu_{1}^{+}-\nu_{2}^{-}}{d_{2}\left(\nu_{1}^{+}, \lambda\right)}-\frac{\nu_{1}^{-}-\nu_{2}^{-}}{d_{2}\left(\nu_{1}^{-}, \lambda\right)}\right) .
\end{aligned}
$$

Having now defined $G_{\lambda}$ to the right of the essential spectrum, extending this function into the essential spectrum is straightforward. One immediately notices that singularities arise for various values of $\lambda$ :

- When $\nu_{1}^{+}(\lambda)=\nu_{1}^{-}(\lambda)$ we find a singularity in $G_{\lambda}^{11}$ and $G_{\lambda}^{21}$. One easily computes that this singularity occurs at,

$$
\lambda=\left(1-a_{1}\right)-\frac{s^{2}}{4 \epsilon^{2}} .
$$

When $s=2 \epsilon \sqrt{1-a_{1}}$ this singularity lies on the imaginary axis. We label this speed $s_{u}$ and it gives the spreading speed of perturbations in the $u$ component. We immediately see that $G_{\lambda}^{11}$, when viewed in this frame is,

$$
G_{\lambda}^{11}(\xi-y)=\left\{\begin{array}{ll}
\frac{1}{\sqrt{\lambda}} e^{\nu_{1}^{-}(\lambda)(\xi-y)} & \text { for } \quad \xi>y \\
\frac{1}{\sqrt{\lambda}} e^{\nu_{1}^{+}(\lambda)(\xi-y)} & \text { for } \xi<y
\end{array} .\right.
$$

Notice the lack of analyticity about $\lambda=0$. A similar non-analytic singularity occurs in $G_{\lambda}^{22}$, but since it is always confined to the stable half-plane we do not remark on it further. 
- Next, we note that whenever $d_{1}\left(\nu_{1}^{ \pm}(\lambda), \lambda\right)=0$, there exists a singularity in $G_{\lambda}^{21}$. We focus on the case when $d_{1}\left(\nu_{1}^{-}(\lambda), \lambda\right)=0$ for some value of $\lambda$. This implies that either $\nu_{1}^{-}(\lambda)=\nu_{2}^{-}(\lambda)$ or $\nu_{1}^{-}(\lambda)=\nu_{2}^{+}(\lambda)$. We consider the case of $\nu_{1}^{-}=\nu_{2}^{+}$. We note first that these roots are analytic functions of $\lambda$ provided that $\lambda$ is to the right of the branch point formed when $\nu_{1}^{+}=\nu_{1}^{-}$. This is due to the fact that both these roots are analytic solutions of the individual dispersion relations $d_{1}(\nu, \lambda)$ and $d_{2}(\nu, \lambda)$. The full dispersion relation is just the product of these two, so $\nu_{1}^{-}$and $\nu_{2}^{+}$remain analytic roots of the full equation.

Let $\lambda^{*}$ be so that $\nu_{1}^{-}\left(\lambda^{*}\right)=\nu_{2}^{+}\left(\lambda^{*}\right)$. Consider $\xi>y$. In this case, we note that $c_{1}(\lambda)$ has a removable singularity at $\lambda^{*}$. However, the second term in (4.4) is unbounded in a neighborhood of $\lambda^{*}$ and this point is observed to be a pole of $G_{\lambda}^{21}$. For $\xi<y$, this pole singularity remains in the coefficient $c_{2}(\lambda)$.

The spreading speed associated to this pole is found by solving for the value of $s$ that places this double root on the imaginary axis. We find,

$$
s_{\text {lin }}=\frac{1-a_{1}}{\sqrt{1+r-a_{1}}}+\mathcal{O}(\epsilon)
$$

We denote this speed as the linear spreading speed. Notice the order in magnitude difference in the size of $s_{l i n}, s_{u}$ and $\epsilon \sigma_{c r i t}$. According to the argument above, we then expect a meromorphic extension of the Green's function into the left half plane.

Before proceeding, we remark that one might expect a pole to exist at the value of $\lambda$ for which $\nu_{1}^{-}(\lambda)=\nu_{2}^{-}(\lambda)$. This could be troubling, for we can not exclude the possibility that this alignment of eigenvalues occurs to the right of the essential spectrum, where we know that $G_{\lambda}$ is analytic and the operator invertible. The resolution is to note that this point is a removable singularity. Indeed, for $\xi>y$ the function $G_{\lambda}^{21}$ has singularities in both $c_{1}(\lambda)$ as well as the second term in (4.4). Nonetheless, these singularities compensate for one another so that this function remains bounded on a neighborhood of the singularity. As a result, this singularity is removable and an analytic extension of $G_{\lambda}$ to this point is available.

We make some remarks before turning our attention to the nonlinear traveling front.

Remark 3. The physical mechanism leading to this fast linear spreading in the linearized equations can be understood by a formal analysis of (4.1). For some small, localized perturbation of the fixed point $P_{-}$, the $p$ component grows exponentially and diffuses slowly. This exponential growth acts as a source term in the $q$ equation, driving the growth of $q$, which in turn diffuses quickly. The $\mathcal{O}(1)$ diffusion constant leads to $\mathcal{O}(1)$ spreading in the $q$ equation.

In order to see the basic mechanism, we set $\epsilon=0$ and consider $\delta$-distribution initial conditions, $p(0, x)=$ $\delta(x)$. In this case, $p(t, x)=e^{\left(1-a_{1}\right) t} \delta(x)$. Substituting into (4.1), we find an equation for the evolution of $q$,

$$
q_{t}=q_{x x}-r q-r a_{2} e^{\left(1-a_{1}\right) t} \delta(x) .
$$

Let $w=e^{-\left(1-a_{1}\right) t} q$, then

$$
w_{t}=w_{x x}-\left(1-a_{1}+r\right) w-r a_{2} \delta(x) .
$$

The solution of this equation can be expanded as a sum of a homogeneous solution, $w_{0}$ and a particular solution $\tilde{w}$. The particular solution, $\tilde{w}$, satisfies,

$$
0=\tilde{w}_{x x}-\left(1-a_{1}+r\right) \tilde{w}-r a_{2} \delta(x) .
$$

We find $\tilde{w}=C e^{-\sqrt{1-a_{1}+r}|x|}$. This implies that the dynamics of the $q$ equation are,

$$
q(t, x)=e^{\left(1-a_{1}\right) t} \tilde{w}(x)+e^{\left(1-a_{1}\right) t} w_{0}(t, x)=C e^{-\sqrt{1-a_{1}+r}|x|+\left(1-a_{1}\right) t}+\frac{e^{-r t}}{\sqrt{4 \pi t}} \int_{\mathbb{R}} e^{-\frac{(x-y)^{2}}{4 t}} q_{0}(y) d y .
$$

Note that the second, homogeneous term decays pointwise in any frame, but that the particular solution does not. In fact, this term is constant along rays $x=s t$, with $s$ the leading order approximation to the linear spreading speed (4.5). 


\subsection{Stability of the nonlinear front despite the fast linear spreading speed}

We finally come to address the stability of the nonlinear front from Theorem 1 in spite of the faster linear spreading mechanism we just described. We have already established the stability of this front in Theorem 3. The interesting question is how this front can be "pointwise" stable in a frame moving with the selected wavespeed, when on half the real line it appears to be a pointwise unstable state. We first present a formal argument similar to the one in remark 3. We then show that this is not an artifact of this system, but that linear spreading speeds that arise as poles of the pointwise Green's function do not, in general, lead to instabilities of nonlinear front solutions.

Our formal argument proceeds as follows. Take the linear system (4.1) and add a cubic damping term to the $p$ equation. This gives the system,

$$
\begin{aligned}
p_{t} & =\epsilon^{2} p_{x x}+\left(1-a_{1}\right) p-\left(1-a_{1}\right) p^{3} \\
q_{t} & =q_{x x}-r q-r a_{2} p .
\end{aligned}
$$

Again, we consider a $\delta$-distribution initial condition in $p$. Instead of growing exponentially, this perturbation will be quenched into the stable nonlinear state $p=1$, and spread slowly, leading to an approximate solution $p(t, x)=\chi_{\left[-2 \epsilon s_{u} t, 2 \epsilon s_{u} t\right]}$. Consider now the dynamics for the $q$ equation,

$$
q_{t}=q_{x x}-r q-r a_{2} \chi_{\left[-2 \epsilon s_{u} t, 2 \epsilon s_{u} t\right]} .
$$

The dynamics of this equation are simply an adiabatic relaxation to the steady state,

$$
q(t, x)=\left(\partial_{x x}-r\right)^{-1} \chi_{\left[-2 \epsilon s_{u} t, 2 \epsilon s_{u} t\right]} .
$$

This solution is uniformly, exponentially localized about $\left[-2 \epsilon s_{u} t, 2 \epsilon s_{u} t\right]$ so that the spreading speed is $\mathcal{O}\left(\epsilon s_{u}\right)$. This argument suggests that the introduction of a simple nonlinearity can destroy the mechanism for fast growth present in the linear system.

To understand this phenomena in a more general way we again appeal to Green's functions. The linear response of perturbations to the traveling wave is given by equations (3.1). Analogous to the homogeneous case, the response of this system can be expressed in terms of Green's functions. The same general picture holds in this case: to the right of the essential spectrum the pointwise Green's function is a proxy for the resolvent operator and since the linear system is sectorial we know that a contour can be placed to the right of all singularities and the full Green's function, $G(t, x ; y)$ can be found by inverse Laplace Transform. Pointwise decay is achieved if the Green's function can be analytically continued into the left half plane. The obstacles are the same as the homogeneous case: either we find values of $\lambda$ for which $G_{\lambda}$ has poles or we find values where $G_{\lambda}$ has branch poles. In the first case, a meromorphic extension is possible while in the latter no continuation is possible about this point.

Convert the system of second order equations into a system of four first order differential equations, which we write,

$$
\frac{d \phi}{d \xi}=A(\xi, \lambda) \phi, \quad \phi=\left(p, p_{\xi}, q, q_{\xi}\right)^{T}
$$

The only $\xi$ dependence in $A$ is through the nonlinear front, which converges exponentially to its asymptotic rest state. As a result, we have $A(\xi, \lambda) \rightarrow A_{ \pm}(\lambda)$ exponentially. For $\lambda$ to the right of the essential spectrum these asymptotic matrices are hyperbolic. It is well known that one can construct solutions to the nonautonomous system with the same asymptotics. The $(p, q)$ components of these solutions can be used to construct the pointwise Green's function. The relevant solutions are those that are bounded on either halfline, or those that are asymptotic to the stable subspace as $\xi \rightarrow \infty$ and asymptotic to the unstable subspace as $\xi \rightarrow-\infty$.

Extending the Green's function into the essential spectrum is then possible so long as these stable and unstable subspaces can be continued analytically. Of course, $A_{-}(\lambda)$ is just the linearizion about the homogeneous state that we studied in detail in section 4.1. The unstable subspace of this system can be 
extended analytically so long as the unstable eigenvalues do not lose analyticity. These eigenvalues are exactly the roots of the dispersion relation calculated in (4.2). A loss of analyticity occurs only for values of $\lambda$ for which either $\nu_{1}^{+}=\nu_{1}^{-}$or $\nu_{2}^{+}=\nu_{2}^{-}$. As we noted above, the first of these occurs at $\lambda=\left(1-a_{1}\right)-\frac{s^{2}}{4 \epsilon^{2}}$ and corresponds to the spreading speed $s_{u}$. Viewed in a frame moving with the selected wavespeed $\epsilon \sigma_{c r i t}$ this branch point lies in the stable half plane.

As a result, the subspaces necessary for the construction of $G_{\lambda}$ in the case of the linearization about the front can be continued analytically into the left half plane. However, note that this is not related to whether the unstable subspace of $A_{-}$intersects the stable subspace of $A_{-}$for some value of $\lambda$. From the perspective of the Green's function, the stable subspace of $A_{-}$has no influence on the dynamics of the linear system. Therefore, a fast linear spreading induced by a pole of the pointwise Green's function for (4.1) poses no a priori restriction on the spreading speed of the nonlinear system.

\section{Discussion}

To conclude, we make some remarks on general aspects of the slower than linear spreading observed in this paper. In brief, we discuss necessary conditions for the appearance of such phenomena and argue that this behavior should arise in a number of systems of physical interest.

Slower than linear spreading occurs only when the linear spreading speed is induced by a regular pole of the pointwise Green's function, as opposed to a branch pole. For a reaction-diffusion equation with diagonal diffusion matrix, these regular poles arise when the linearization at the unstable state has a block triangular form,

$$
\begin{aligned}
& u_{t}=D_{u} u_{x x}+A u+B v \\
& v_{t}=D_{v} v_{x x}+C v .
\end{aligned}
$$

This block triangular form is enforced by the existence of invariant subspaces for the spatially homogeneous ordinary differential equation, wherein the evolution of some subset of the species decouple at the linear level. The spreading mechanism that emerges due to regular poles of the pointwise Green's function is then analogous to the one in Remark 3.

One immediate consequence of the above discussion is that the selected wavespeed for a system of reactiondiffusion equations is not continuous with respect to system parameters. Consider a small perturbation of parameters that destroys the skew-product form of the linearization. The regular pole requires this specialized structure and therefore can no longer exist after perturbation. On the other hand, any regular pole of $G_{\lambda}$ corresponds to a root of the complex analytic dispersion relation and as such, can not disappear as parameters are varied in an analytic fashion. The resolution is that the regular pole splits into two branch poles which perturb continuously with respect to system parameters. The spreading speeds associated to these branch poles do place a lower bound on the spreading speeds of the full system. Therefore, if the selected wavespeed in the unperturbed case was much slower than the speed associated to the regular pole, upon perturbation we observe a discontinuous jump in the wavespeed.

For an explicit example, consider equation (1.1) with a linear function of $v$ incorporated into the $u$ dynamics,

$$
\begin{aligned}
u_{t} & =\epsilon^{2} u_{x x}+\left(1-u-a_{1} v\right) u+\alpha v \\
v_{t} & =v_{x x}+r\left(1-a_{2} u-v\right) v .
\end{aligned}
$$

For $\alpha \neq 0$, the invariance of the $v$ axis is broken and one can calculate that the regular pole splits into two branch poles and linear spreading speeds in both components are observed to be $\mathcal{O}(1)$, in contrast to the $\mathcal{O}(\epsilon)$ spreading shown in Theorem 1.

Another example of this phenomena is found in the phase field model,

$$
\begin{aligned}
c_{t} & =c_{x x}-e(e-a)(1-e)-\gamma c \\
e_{t} & =\kappa e_{x x}+e(e-a)(1-e)+\gamma c .
\end{aligned}
$$


When $\gamma=0$, the $e$ equation decouples and its dynamics are given by Nagumo's equation, for which a traveling front develops with finite speed. This front acts as a bounded source term in the $c$ equation, leading to spreading of the $c$ component at the same rate. However for some parameter values, one can calculate that there exists a regular pole of the Green's function that leads to faster spreading on the linear level, see [13] for the calculation of this speed. Of course, the nonlinear system does not exhibit spreading at this faster speed, but perturbing $\gamma$ away from zero breaks the skew-product form of the linearizion, splitting this regular pole into two branch poles and a discontinuous jump in the selected nonlinear spreading speed is observed.

That this phenomena of slower than linear spreading depends so crucially on the skew-product structure of the linearization might lead one to dismiss this phenomena as a degenerate case. However, we argue that this structure is an inherent feature of many interesting natural systems. Recall that we are interested in the transition of a nonlinear system away from an unstable homogeneous state. We find it instructive to consider the question of how, in practice, a natural system could attain an unstable homogeneous state in the first place. One of the simplest mechanisms is the one described in this paper: the instability with respect to an invasive species is irrelevant until that species is actually introduced. Unstable states of this form give rise naturally to the necessary skew product structure required for regular poles.

A similar mechanism is observed in pattern forming systems. Consider for instance the Swift-Hohenberg equation,

$$
u_{t}=-(\Delta+1)^{2} u+\epsilon u+\beta|\nabla u|^{2}-u^{3},
$$

in a horizontal strip $(x, y) \in \mathbb{R} \times S^{1}$ with periodic boundary conditions. The set of solutions that is invariant under vertical translations, that is to say solutions with $u(x, y)$ a function of $x$ only, forms an invariant subspace. Equilibria in this subspace may, however, be unstable with respect to perturbations that are not constant in $y$. The most common example would be an instability of horizontal rolls with respect to perturbations that create hexagons.

The analogy between population dynamics and pattern-forming systems becomes more apparent when pattern formation is described by amplitude equations (see for example [7]), that is, the actual independent variable $u(t, x), x \in \mathbb{R}^{2}$ is approximated by complex amplitude vectors $u(t, x) \sim A_{1} e^{i k_{1} \cdot x}+A_{2} e^{i k_{2} \cdot x}$. The amplitudes $A_{j}$ then evolve on slow time scales according to coupled mode equations,

$$
\begin{aligned}
& \left(A_{1}\right)_{t}=d_{1}\left(A_{1}\right)_{x x}+\mu_{1} A_{1}-A_{1}\left(\left|A_{1}\right|^{2}+a_{1}\left|A_{2}\right|^{2}\right) \\
& \left(A_{2}\right)_{t}=d_{2}\left(A_{2}\right)_{x x}+\mu_{2} A_{2}-A_{2}\left(\left|A_{2}\right|^{2}+a_{2}\left|A_{1}\right|^{2}\right) .
\end{aligned}
$$

When effective diffusivities satisfy $d_{1} / d_{2} \ll 1$ and mutual mode inhibition parameters $a_{1}, a_{2}$ satisfy $a_{1}<1$ and $a_{2}>1$, the competition between $A_{1}$ and $A_{2}$ is described by invasion fronts in a completely analogous fashion to the Lotka-Volterra competition system. It is worth noticing that the presence of an invariant subspace here may well be caused by normal form (or averaging) symmetries that arise when performing the amplitude expansion, rather than an obvious invariance condition as outlined in the Swift-Hohenberg case above.

In conclusion, the example in this paper demonstrates that wavespeed selection mechanisms for systems of reaction-diffusion equations can differ significantly from the selection mechanisms familiar for scalar systems. We expect that a characterization of these new modes of invasion will be a fruitful line of future research.

\section{Acknowledgments}

The research of MH was supported by the NSF (DMS-1004517). The research of AS was supported by the NSF (DMS-0806614).

\section{References}

[1] D. G. Aronson and H. F. Weinberger. Multidimensional nonlinear diffusion arising in population genetics. Adv. in Math., 30(1):33-76, 1978. 
[2] A. Bers. Space-time evolution of plasma instabilities-absolute and convective. In A. A. Galeev \& R. N. Sudan, editor, Basic Plasma Physics: Selected Chapters, Handbook of Plasma Physics, Volume 1, pages 451-517, 1984.

[3] L. Brevdo. A study of absolute and convective instabilities with an application to the eady model. Geophysical \&3 Astrophysical Fluid Dynamics, 40:1-92, 1988.

[4] R. J. Briggs. Electron-Stream Interaction with Plasmas. MIT Press, Cambridge, 1964.

[5] J. Carr. Applications of centre manifold theory, volume 35 of Applied Mathematical Sciences. SpringerVerlag, New York, 1981.

[6] S.-N. Chow, C. Z. Li, and D. Wang. Normal forms and bifurcation of planar vector fields. Cambridge University Press, Cambridge, 1994.

[7] M. C. Cross and P. C. Hohenberg. Pattern formation outside of equilibrium. Rev. Mod. Phys., 65:8511112, Jul 1993.

[8] G. Dee and J. S. Langer. Propagating pattern selection. Phys. Rev. Lett., 50(6):383-386, Feb 1983.

[9] R. Derndinger. Uber das spektrum positiver generatoren. Mathematische Zeitschrift, 172:281-293, 1980.

[10] U. Ebert and W. van Saarloos. Front propagation into unstable states: universal algebraic convergence towards uniformly translating pulled fronts. Phys. D, 146(1-4):1-99, 2000.

[11] N. Fenichel. Geometric singular perturbation theory for ordinary differential equations. J. Differential Equations, 31(1):53-98, 1979.

[12] R. A. Fisher. The wave of advance of advantageous genes. Annals of Human Genetics, 7(4):355-369, 1937.

[13] R. N. Goh, S. Mesuro, and A. Scheel. Spatial wavenumber selection in recurrent precipitation. SIAM Journal on Applied Dynamical Systems, 10:360-402, 2011.

[14] J.-S. Guo and X. Liang. The minimal speed of traveling fronts for the Lotka-Volterra competition system. Journal of Dynamics and Differential Equations, 23:353-363, 2011.

[15] D. Henry. Geometric theory of semilinear parabolic equations, volume 840 of Lecture Notes in Mathematics. Springer-Verlag, Berlin, 1981.

[16] Y. Hosono. The minimal speed of traveling fronts for a diffusive Lotka-Volterra competition model. Bulletin of Mathematical Biology, 60:435-448, 1998.

[17] Y. Hosono. Traveling waves for a diffusive Lotka-Volterra competition model. I. Singular perturbations. Discrete Contin. Dyn. Syst. Ser. B, 3(1):79-95, 2003.

[18] W. Huang. Problem on minimum wave speed for a Lotka-Volterra reaction-diffusion competition model. Journal of Dynamics and Differential Equations, 22:285-297, 2010.

[19] P. Huerre and P. A. Monkewitz. Local and global instabilities in spatially developing flows. Annual Review of Fluid Mechanics, 22:473-537, 1990.

[20] C. K. R. T. Jones. Geometric singular perturbation theory. In Dynamical systems (Montecatini Terme, 1994), volume 1609 of Lecture Notes in Math., pages 44-118. Springer, Berlin, 1995.

[21] Y. Kan-On and Q. Fang. Stability of monotone travelling waves for competition-diffusion equations. Japan Journal of Industrial and Applied Mathematics, 13:343-349, 1996. 
[22] Y. Kan-on and E. Yanagida. Existence of nonconstant stable equilibria in competition-diffusion equations. Hiroshima Math. J., 23(1):193-221, 1993.

[23] A. Kolmogorov, I. Petrovskii, and N. Piscounov. Etude de l'equation de la diffusion avec croissance de la quantite' de matiere et son application a un probleme biologique. Moscow Univ. Math. Bull., 1:1-25, 1937.

[24] M. Krupa and P. Szmolyan. Extending geometric singular perturbation theory to nonhyperbolic pointsfold and canard points in two dimensions. SIAM J. Math. Anal., 33(2):286-314, 2001.

[25] M. A. Lewis, B. Li, and H. F. Weinberger. Spreading speed and linear determinacy for two-species competition models. J. Math. Biol., 45(3):219-233, 2002.

[26] B. Li, H. F. Weinberger, and M. A. Lewis. Spreading speeds as slowest wave speeds for cooperative systems. Math. Biosci., 196(1):82-98, 2005.

[27] B. Sandstede. Stability of travelling waves. In Handbook of dynamical systems, Vol. 2, pages 983-1055. North-Holland, Amsterdam, 2002.

[28] B. Sandstede and A. Scheel. Absolute and convective instabilities of waves on unbounded and large bounded domains. Phys. D, 145(3-4):233-277, 2000.

[29] W. van Saarloos. Front propagation into unstable states. Physics Reports, 386(2-6):29 - 222, 2003. 